\title{
Atemwegsmanagement im Rettungsdienst
}

Mark Michael, Juliane Tautz, Frank Sensen, Michael Bernhard

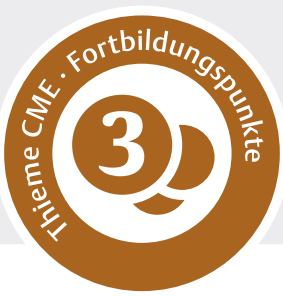

Im Einsatzspektrum des professionellen Rettungsdienstes stellen Atemwegsnotfälle („A-Probleme“) einen seltenen, respiratorische Notfälle („B-Probleme“) hingegen einen häufigen Einsatzgrund dar. Die Vorhaltung entsprechenden Materials, Schulung und regelmäßiges Training, die Etablierung von SOPs und gelebtes Teamwork sind wesentliche Aspekte für ein erfolgreiches Atemwegsmanagement, für das die S1-Leitlinie „Prähospitales Atemwegsmanagement“ einen praktischen Leitfaden bietet.

\section{Einleitung}

Das Atemwegsmanagement nimmt einen zentralen Anteil der prähospitalen Notfallversorgung ein. Nicht nur die Sauerstoffgabe, sondern auch das nichtinvasive und invasive Atemwegsmanagement sind bei zahlreichen Notfallbildern relevanter Bestandteil der Therapie. Zen- trale Herausforderung sind eine adäquate Oxygenierung und Ventilation als Grundlage für alle weiteren Maßnahmen der prähospitalen Versorgung.

Atemwegsverlegungen treten bei einer Vielzahl an Notfallsituationen auf, z. B. anaphylaktische Reaktionen, Vigilanzminderung, Aspiration/Bolusgeschehen. Zudem stel-

\begin{tabular}{|c|c|c|c|}
\hline \multicolumn{4}{|c|}{ ABKÜRZUNGEN } \\
\hline \multirow[t]{2}{*}{ (c)ABCDE } & (Critical Bleeding) - Airway - Breathing - & FONA & Front-of-Neck Access \\
\hline & Circulation - Disability - Exposure/Examination & GCS & Glasgow Coma Scale \\
\hline ACCD & Automated Chest Compression Device & HWS & Halswirbelsäule \\
\hline $\mathrm{AF}$ & Atemfrequenz & ID & Innendurchmesser \\
\hline ÄLRD & Ärztlicher Leiter Rettungsdienst & ILCOR & International Liaison Committee \\
\hline BMV & Beutel-Masken-Beatmung & & on Resuscitation \\
\hline \multirow[t]{2}{*}{ BURP-Manöver } & Backward Upward Rightward Pressure & MILS & manuelle Inline-Stabilisierung \\
\hline & (Druck nach hinten oben rechts) & MNS & Mund-Nasen-Schutz \\
\hline $\mathrm{CICO}$ & Cannot intubate, cannot oxygenate & NEF & Notarzteinsatzfahrzeug \\
\hline COPD & chronisch obstruktive Lungenerkrankung & NIBP & Non-invasive Blood Pressure \\
\hline COVID-19 & Corona Virus Disease 2019 & NIV & nichtinvasive Beatmung \\
\hline CPAP & Continuous Positive Airway Pressure & $\mathrm{OHCA}$ & Out of Hospital cardiac Arrest \\
\hline CPR & Cardiopulmonary Resuscitation & PCV & Pressure controlled Ventilation \\
\hline \multirow[t]{2}{*}{ DGAI } & Deutsche Gesellschaft für Anästhesiologie & PEEP & positive endexpiratory Pressure \\
\hline & und Intensivmedizin & $P_{\text {insp }}$ & Inspirationsdruck \\
\hline \multirow[t]{4}{*}{ DOPES } & Dislokation des Tubus - Obstruktion - & PSA & persönliche Schutzausrüstung \\
\hline & Pneumothorax/andere pulmonale Störungen - & Rekap & Rekapillarisierungszeit \\
\hline & Equipmentversagen - Stomach (Magenüber- & ROSC & Return of spontaneous Circulation \\
\hline & blähung)/Spezielles & RR & Blutdruck \\
\hline EGA & extraglottischer Atemweg & RSI & Rapid Sequence Induction \\
\hline etCO & endtidales Kohlendioxid & RTW & Rettungstransportwagen \\
\hline ETI & endotracheale Intubation & SAA & Standard-Arbeitsanweisung \\
\hline ETT & Endotrachealtubus & SOP & Standard Operating Procedure \\
\hline FFP2 & Filtering Face Piece (Klasse 2) & & \\
\hline $\mathrm{FiO}_{2}$ & inspiratorische Sauerstofffraktion & & \\
\hline
\end{tabular}


len Notfälle mit einer respiratorischen Insuffizienz und damit Oxygenierungs- und/oder Ventilationsstörungen einen Großteil der rettungsdienstlichen Versorgung dar [1].

Grundzüge des prähospitalen Atemwegsmanagements sind

- das Freihalten der Atemwege („A“ = Atemweg/Airway) mit Basismaßnahmen wie dem mechanischen Freimachen und Freihalten der Atemwege und erweiterte Techniken,

- Sicherung einer ausreichenden Oxygenierung („B“ = Be-/Atmung/Breathing) sowie eines Aspirationsschutzes,

- insbesondere bei persistierender Vigilanzminderung („D“ = Vigilanzstörungen/Disability).

Merke

Das prähospitale Atemwegsmanagement ist eine interprofessionelle Herausforderung für das gesamte Rettungsteam. Vor diesem Hintergrund müssen sowohl das nichtärztliche Personal als auch Notärzte mit den Grundlagen und den erweiterten Maßnahmen zur Aufrechterhaltung eines freien Atemwegs und der Oxygenierung und Ventilation vertraut sein [1].

Das nichtärztliche Rettungsdienstpersonal (z. B. Rettungssanitäter, Rettungsassistenten, Notfallsanitäter) erlernt im Rahmen der Ausbildung neben Grundlagen der Atemwegssicherung zum Teil auch Maßnahmen der nichtinvasiven Beatmung (NIV) und der nichtinvasiven und invasiven Atemwegssicherung (z. B. extraglottische Atemwegssicherung [EGA]: Larynxmaske, Larynxtubus bei Reanimationen); diese Maßnahmen werden in definierten Situationen im Rahmen der Delegation ausgeübt.

Die nichtinvasive Ventilation ist in den letzten Jahren bei der Versorgung der respiratorischen Insuffizienz zu einem festen Bestandteil der prähospitalen Therapie geworden. Die invasive Atemwegssicherung insbesondere bei Patienten außerhalb von Reanimationssituationen stellt nach wie vor eine originäre notärztliche Maßnahme dar, und es existieren Leitlinien zum Vorgehen [1,2].

\section{Leitlinie prähospitales}

\section{Atemwegsmanagement}

Die im Jahre 2019 zuletzt aktualisierte S1-Leitlinie „Prähospitales Atemwegsmanagement“ wurde von einem Expertengremium der Deutschen Gesellschaft für Anästhesiologie und Intensivmedizin (DGAI) grundlegend überarbeitet und aktuellen Entwicklungen angepasst [1]. Es gelten 10 wesentliche „Soll-Empfehlungen“ (s. Übersicht), und ein strukturierter Algorithmus bietet einen praxisnahen Leitfaden zu allen Aspekten der prähospita-

\section{ÜBERSICHT}

Die zentralen „Soll“-Empfehlungen zur Sicherung der Atemwege in der Notfallmedizin

1. Kritische Überprüfung der Indikation zur invasiven Atemwegssicherung.

2. Obligate Durchführung einer adäquaten Präoxygenierung mit höchstmöglicher $\mathrm{FiO}_{2}$ vor invasiver Atemwegssicherung (ETI oder EGA) beim spontanatmenden Patienten.

3. Endotracheale Intubation als Goldstandard der invasiven Atemwegssicherung beim Erwachsenen, jedoch nur, wenn mindestens $100 \mathrm{ETI}$ an Patienten unter Aufsicht dokumentiert und $10 \mathrm{ETI} /$ Jahr durchgeführt wurden.

4. EGA als primärer Atemweg, wenn o. g. Anforderungen nicht erfüllt werden können und wenn 45 Anwendungen unter Aufsicht dokumentiert und 3 Anwendungen/Jahr durchgeführt wurden oder als Alternative bei schwieriger Intubation.

5. Verwendung von EGA mit Drainagekanal und Platzieren einer Magensonde oder einer EGA mit hoher Erfolgswahrscheinlichkeit einer anschließenden, nicht optisch geführten Intubation.

6. Primärer Einsatz der Videolaryngoskopie zur endotrachealen Intubation mit Macintoshähnlichem Spatel.

7. Als primäre Technik zur Beatmung von Kindern soll die optimierte Maskenbeatmung mit beidhändigem Esmarch-Maskengriff (doppelter C-Griff), optimaler Kopflagerung \pm passender Guedel-Tubus und mit einer hohen $\mathrm{FiO}_{2}$ mit Beutel-Maske-Beatmung durchgeführt werden.

8. Als extraglottischer Atemweg wird bei Kindern die Larynxmaske empfohlen.

9. Kontinuierliche Kapnografie nach jeder Atemwegssicherungsmaßnahme.

10. Ausbildung der Techniken am Patienten ist ausnahmslos notwendig, Übungen am Phantom allein ist nicht ausreichend. Es muss eine Anpassung der prähospitalen Ausstattung, insbesondere bei den EGA und der Videolaryngoskopie, auf die innerklinische Ausbildung stattfinden.

(modifiziert nach [1])

EGA = extraglottische Atemwegshilfe, ETI = endotracheale Intubation, $\mathrm{FiO}_{2}=$ inspiratorische Sauerstofffraktion

len Atemwegssicherung bei Kindern und Erwachsenen ( A Abb. 1). Aufgrund der außerordentlichen Relevanz der Leitlinie und des Bezugs zum bundesdeutschen Rettungsdienst orientiert sich auch dieser Übersichtsartikel maßgeblich an den Grundaussagen dieser Leitlinie. 


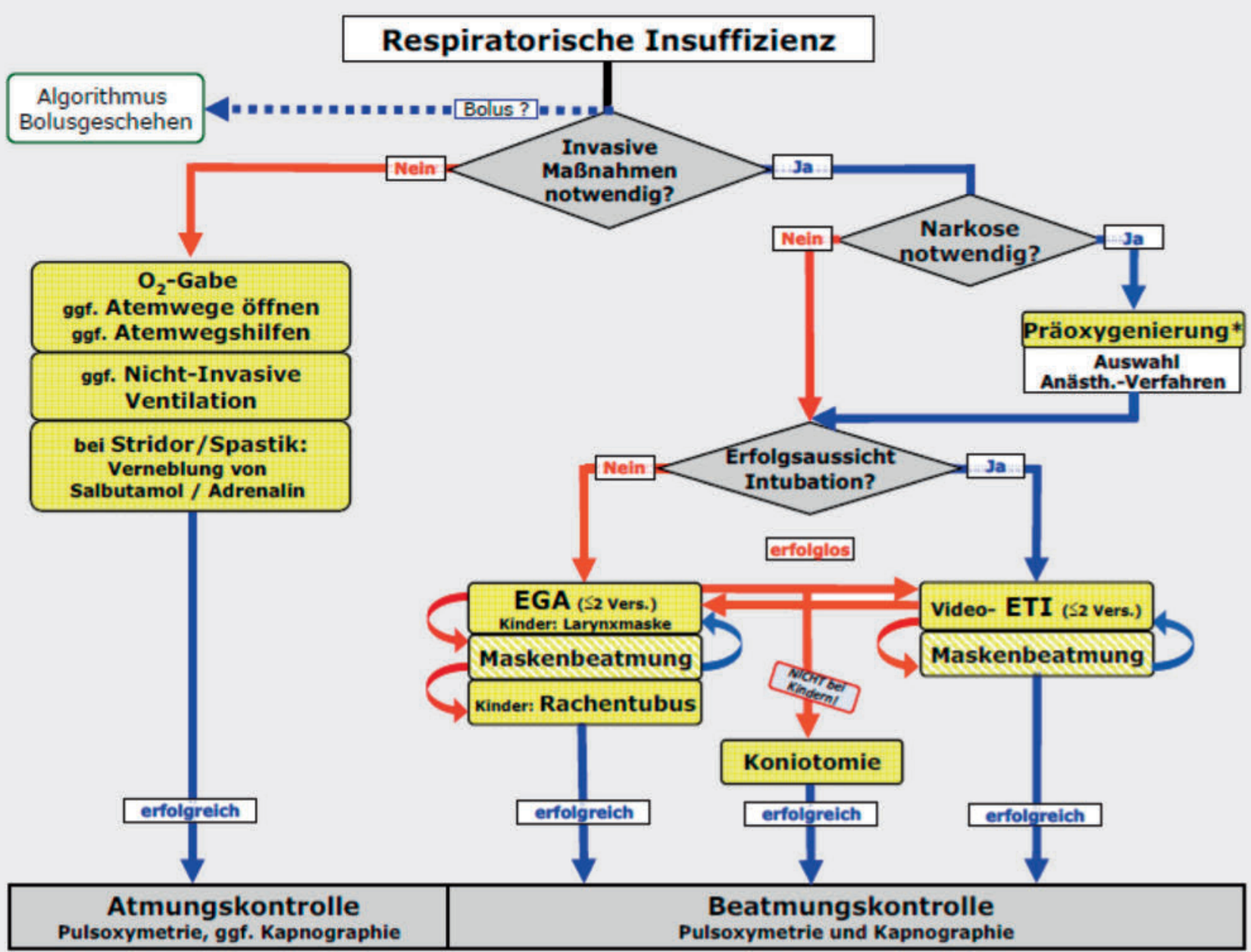

Abb. 1 Algorithmus zum prähospitalen Atemwegsmanagement. (Quelle: Timmermann A, Böttiger BW, Byhahn C et al. S1-Leitlinie: Prähospitales Atemwegsmanagement. AWMF Online 2019. AWMF-Registernummer 001-040. Im Internet (Stand: 27.10.2020): https://www.awmf.org/leitlinien/ detail/II/001-040.html) [rerif]

\section{FALLBEISPIEL}

Das Rettungsteam (RTW und NEF) wird zu einem 55-jährigen Patienten gerufen, der im Badezimmer auf der Toilette synkopiert ist. Die Alarmierung erfolgte durch die Ehefrau, die durch einen dumpfen Aufprall auf die Situation aufmerksam wurde. Beide Fahrzeuge treffen etwa 8 Minuten nach Alarmierung parallel am Einsatzort ein und finden den Patienten nicht ansprechbar auf dem Badezimmerboden liegend vor.

Beim Primary Survey zeigt der Patient zunächst keine Reaktion auf die Ansprache des Rettungsteams. Ein „A-Problem“ im Sinne einer drohenden Atemwegsverlegung imponiert durch ein „schnarchendes Atemgeräusch“. Durch den sofortigen Einsatz des Esmarch-Handgriffs können die oberen Atemwege zunächst freigehalten werden.

Die weiteren Untersuchungsergebnisse ergeben:

- B - Breathing: Zyanose, Bradypnoe 8/min, $\mathrm{SpO}_{2} 90 \%$ unter Raumluft.
- C - Circulation: Rekap 2 s, kräftige periphere Pulse seitengleich palpabel, Hypertension mit RR 190/100 mmHg, keine Blutungszeichen.

- D - Disability: Vigilanzminderung mit GCS 3 (keinerlei Reaktion auf Schmerzreize und Ansprache), Anisokorie links > rechts.

- E - Environment: Normothermie $36,6^{\circ} \mathrm{C}$, im Bodycheck keine Verletzungszeichen eruierbar, insbesondere keine Kopfverletzungen.

Das Rettungsteam entscheidet sich zunächst aufgrund eines möglichen Traumamechanismus für eine HWS-Immobilisation (A, C), die Sauerstoffgabe via Inhalationsmaske und Reservoir mit einem Flow von $8 \mathrm{l} / \mathrm{min}$ (B), die Anlage eines periphervenösen Zugangs am Unterarm rechts, 17 Gauge (C) und die obligate Blutzuckermessung (D), die einen normwertigen Befund von $95 \mathrm{mg} / \mathrm{dl}$ ergibt. 


\section{Standard-Arbeitsanweisungen für Rettungsdienstpersonal}

Rettungssanitäter, Rettungsassistenten und Notfallsanitäter erlernen im Rahmen der klinischen Ausbildung Grundlagen der Atemwegssicherung. Insbesondere für Notfallsanitäter ist im Rahmen der Delegation auch die Durchführung ärztlicher Maßnahmen vorgesehen, um das therapiefreie Intervall zu verkürzen. So sind in standardisierten Handlungsanweisungen (Standard-Arbeitsanweisungen, SAA; Standard Operating Procedures, SOP) zum Teil auch Maßnahmen der nichtinvasiven Beatmung (NIV) und der invasiven Atemwegssicherung vorgesehen, die in klar definierten Situationen ausgeübt werden können [3]. Diese Maßnahmen sind in Deutschland teilweise auf Ebene der Bundesländer geregelt, im Wesentlichen aber auf kommunaler Ebene verankert und durch den jeweiligen Ärztlichen Leiter Rettungsdienst (ÄLRD) für jeden Rettungsdienstbereich gesondert vorgegeben. Die Maßnahmen werden vielerorts in jährlichen Pflichtfortbildungen gelehrt und geprüft.

Auch wenn es zahlreiche Übereinstimmungen gibt, liegen doch bundesweit keine einheitlichen SAA/SOP vor, obwohl im Rahmen der Notfallsanitäterausbildung ein Maßnahmenkatalog auch im Rahmen des Atemwegsmanagements geschult wird. Gemeinsame, bundesländerübergreifende SAA für Notfallsanitäter existieren z. B. in Mecklenburg-Vorpommern, Sachsen, Sachsen-Anhalt und Nordrhein-Westfalen. Hierin werden für die Ausbildung und Prüfung auch invasive Maßnahmen beschrieben. Im Bereich des Atemwegsmanagements werden das endotracheale Absaugen, der Umgang mit einer Trachealkanüle, die Etablierung einer EGA, die Fremdkörperentfernung mithilfe von Laryngoskop und Magill-Zange, die Entlastungspunktion beim Spannungspneumothorax oder die medikamentöse Therapie z. B. bei Bronchospasmus definiert $[3,4]$. Im prähospitalen Alltag kann so das therapiefreie Intervall bis zum Eintreffen des Notarztes verkürzt werden.

\section{Notärztliche Ausbildung und Expertise}

Die prähospitale Narkoseeinleitung und Atemwegssicherung ist eine ärztliche Kernkompetenz und stellt eine Notarztindikation dar [5]. Im Rahmen der Zusatzweiterbildung „Notfallmedizin“ werden neben der klinischen Expertise (Intensivmedizin, Anästhesie) in den meisten Ärztekammern 25 endotracheale Intubationen als Nachweis gefordert [6]. In der S1-Leitlinie „Prähospitales Atemwegsmanagement" der DGAI wird empfohlen, die Intubation prähospital nur anzuwenden, wenn am Patienten mindestens 100 Intubationen initial sowie nachfolgend mindestens 10 endotracheale Intubationen pro Jahr durchgeführt werden. Die Einschätzung der Erfolgswahrscheinlichkeit der Atemwegssicherung rückt so in den Vordergrund, und so kann im Einzelfall bei geringer Expertise primär eine extraglottische Atemwegssicherung erfolgen [1]

Merke

Die endotracheale Intubation gilt weiterhin als Goldstandard der Atemwegssicherung in Notfallsituationen $[1,7]$. Die fundierte Ausbildung von (angehenden) Notärzten in der Atemwegssicherung sowie das regelmäßige Training bzw. eine entsprechende Expertise der Intubation sollten daher angestrebt werden, um in Notfallsituationen eine adäquate und sichere Atemwegssicherung durchzuführen [8].

Auch für die supraglottische Atemwegssicherung wird die Einlage von mindestens 45 Devices am Patienten unter kontrollierten Bedingungen empfohlen, ein alleiniges Training am Übungsphantom wird als nicht ausreichend erachtet [1]. Des Weiteren ist gefordert, dass Ärztinnen und Ärzte, die regelmäßig am Notarztdienst teilnehmen, nicht nur die klinische Erfahrung im Atemwegsmanagement aufrechterhalten, sondern auch regelmäßig an Fortbildungen zum Themenkomplex teilnehmen [1].

\section{TAKE HOME MESSAGE}

Die endotracheale Intubation gilt als Goldstandard bei der prähospitalen Atemwegssicherung. Abhängig von der Erfolgswahrscheinlichkeit und der Expertise des Teams ist allerdings auch eine supraglottische Atemwegssicherung zu erwägen.

\section{Grundzüge des}

\section{Atemwegsmanagements}

Ziele des prähospitalen Atemwegsmanagements sind, eine situationsangepasste Sicherung der Atemwege sowie eine ausreichende Oxygenierung und Ventilation zu gewährleisten [1,7]. Eine invasive Atemwegssicherung ist dazu meist nur in bestimmten Fällen erforderlich, und so sollte die Indikation grundsätzlich streng und in Abstimmung mit dem gesamten Team erfolgen.

Wie in allen Notfallsituationen üblich wird im Rahmen des Primary Survey der Akutversorgung nach dem ABCDESchema gehandelt, wobei das „A“ und „B“ die respiratorische Situation widerspiegelt: 


\section{A - Atemwege/Airway}

Beurteilung: Atemwege frei? Sekretverlegung? Mechanische Verlegung?

Maßnahmen: Freimachen der Atemwege (EsmarchHandgriff, Oropharyngealtuben), Absaugung, Fremdkörperentfernung (Laryngoskopie/Magill-Zange), endotracheale Intubation.

\section{B - (Be-)Atmung/Breathing}

Beurteilung: suffiziente Oxygenierung und Ventilation? Atemfrequenz? Zyanose? Atemarbeit?

Maßnahmen: Sauerstoffgabe, nichtinvasive Ventilation, invasive Atemwegssicherung, medikamentöse Therapie, Entlastungspunktion bei Spannungspneumothorax.

\section{Basismaßnahmen des Atemwegsmanage- ments}

Basismaßnahmen des Atemwegsmanagements sind zunächst das Freimachen und Freihalten der Atemwege bei einer drohenden oder manifesten Verlegung („A-Problem"). Dies erfolgt im Rahmen des Primary Survey und mithilfe mechanischer Maßnahmen wie dem EsmarchHandgriff, dem Chin-Lift-Manöver oder dem Einsatz von Oropharyngealtuben (Guedel-Tubus, Wendl-Tubus).

Bei einer Atemwegsverlegung durch Fremdkörper sind das orotracheale Absaugen sowie die Fremdkörperentfernung mithilfe von Laryngoskopie und Einsatz der MagillZange etabliert und werden auch als Maßnahmen für Rettungsdienstfachpersonal empfohlen [1,5].

Zur Sicherung einer adäquaten Oxygenierung bzw. Präoxygenierung ist die frühzeitige und - situationsangepasste - hochdosierte Sauerstoffgabe der nächste Schritt der prähospitalen Versorgung. Die Nutzung einer nasalen Sauerstoffbrille (Flow 2-4 I/min) ist als supportiv anzusehen, effektiver ist eine Inhalation über eine Maske mit Reservoir (Flow > 6l/min bis $15 \mathrm{l} / \mathrm{min}$ ), um eine möglichst hohe inspiratorische Sauerstoffkonzentration $\left(\mathrm{FiO}_{2}\right) \mathrm{zu}$ erzielen.

\footnotetext{
Merke

Vor jeglicher invasiven Atemwegssicherung ist über dieses Verfahren, alternativ als nichtinvasive Beatmung (NIV) eine Präoxygenierung sicherzustellen $[1,7]$.
}

In ausgewählten Situationen ist ggf. Zurückhaltung bei der Sauerstoffgabe angezeigt (z.B. chronische Hypoxie bei hochgradiger COPD, Patienten mit akutem Myokardinfarkt) [9]. Wesentlicher Surrogatparameter einer ausreichenden Oxygenierung ist hierbei eine adäquate pulsoxymetrische Sauerstoffsättigung $\left(\mathrm{SpO}_{2}\right)$.

\section{FALLBEISPIEL}

\section{Fortsetzung}

Nach Durchführung des Primary Survey wird ein „A-Problem“ erfolgreich behandelt. Bei persistierender Vigilanzminderung und führendem „D-Problem“ bei GCS 3 und dringendem Verdacht auf das Vorliegen eines ischämischen Insultes oder einer intrakraniellen Blutung wird die Indikation zur dringlichen prähospitalen Atemwegssicherung gestellt und im Team das Prozedere besprochen: Unter Präoxygenierung wird der Patient bei suffizienter Spontanatmung und stabiler Hämodynamik unter Immobilisation (HWSOrthese, Spineboard) wenige Meter in den RTW verbracht, wo ein Teammitglied bereits die Narkoseeinleitung und endotracheale Intubation vorbereitet. Der Notarzt legt die Einleitungsmedikation (Propofol 1\%, Rocuronium, Fentanyl, Norepinephrin) sowie das erforderliche Material (ETT 8,0 mm ID sowie 7,5 mm ID mit Führungsstab, Videolaryngoskop mit Macintosh-Spatel Größe 3, Eschmann-Stab, Absaugung, alternative Atemwegssicherung: Larynxtubus Größe 4 und 5, Koniotomieset) fest.

Der Patient wird in den RTW verbracht und dort auf der Trage gelagert, die Präoxygenierung wird mit einem Sauerstoff-Flow von $15 \mathrm{l} / \mathrm{min}$ via Inhalationsmaske und Reservoirbeutel unter Absaugbereitschaft fortgeführt und nochmals das genaue Prozedere zur geplanten Atemwegssicherung durchgesprochen, zudem die Aufgaben im Team verteilt. Die Indikation zur invasiven Atemwegssicherung in Abwägung der Transportzeit (ca. 20 Minuten zur geeigneten Klinik) wird noch einmal bestätigt. Erneut werden die Atemwege des Patienten inspiziert, hier sind keine anatomischen Auffälligkeiten sichtbar, auch anamnestisch gibt es keine Hinweise auf eine erschwerte Atemwegssicherung.

\section{Nichtinvasive Beatmung}

In den letzten Jahren hat sich die nichtinvasive Ventilation (NIV) zunehmend in der prähospitalen Notfallmedizin durchgesetzt. Die materielle Ausstattung ist mittlerweile meist ubiquitär verfügbar, und sowohl die CPAP-Therapie als auch die NIV-Therapie mit inspiratorischer Druckunterstützung bei Ventilationsstörungen sind in die Behandlungsalgorithmen für Notfallsanitäter integriert [1, 10] (s.a. Infobox).

Durch den frühzeitigen prähospitalen Therapiebeginn sollen eine weitere Dekompensation, aber auch konsekutive invasive Atemwegssicherungen mit entsprechenden Komplikationen vermieden werden [10]. Zudem ist die Therapie einfach durchführbar, und unter Beachtung der Kontraindikationen (z. B. Vigilanzminderung, Aspirationsgefahr) ist eine sichere Anwendung möglich und meist ein rasch einsetzender Effekt zu erzielen. Auch ein effektives „Bridging“ bis zur definitiven Atemwegssicherung unter optimalen klinischen Bedingungen kann durch die NIV-Therapie erreicht werden. Allerdings sollten auch prähospital Abbruchkritierien beachtet werden, 


\section{HINTERGRUNDWISSEN}

\section{Beatmung im Rettungsdienst}

- CPAP-Therapie (z. B. mittels Boussignac-Ventil oder Transportbeatmungsgerät) vor allem bei Oxygenierungsstörungen mittels Anwendung eines PEEP (positiv-endexspiratorischer Druck; Einsatz z. B. beim kardialen Lungenödem, Pneumonie)

- NIV-Therapie mit inspiratorischer Druckunterstützung bei Ventilationsstörungen (z. B. bei exazerbierter COPD)

und es muss ggf. sekundär eine invasive Atemwegssicherung erfolgen $[1,10]$.

Kontraindikationen zur Durchführung einer NIV-Therapie sind unter anderem eine Vigilanzminderung und fehlende Schutzreflexe, mangelnde Compliance, hämodynamische Instabilität oder Aspirationsgefahr [10]. Essenziell ist neben der schrittweisen Durchführung und Anleitung des Patienten das Monitoring der $\mathrm{SpO}_{2}$, der Atemfrequenz, ggf. des et $\mathrm{CO}_{2}$, der Hämodynamik und die klinische Beurteilung der Atemmechanik. Eine unterstützende Analgosedierung, z. B. mit Morphin, kann die Compliance deutlich verbessern und die subjektive Dyspnoe des Patienten reduzieren.

\section{TAKE HOME MESSAGE}

Die nichtinvasive Ventilation (NIV) stellt eine einfache und effektive Maßnahme auch in der prähospitalen Notfallmedizin dar. Bei primären Oxygenierungsstörungen sind die Anwendung eines PEEP, bei Ventilationsstörungen die NIV-Therapie mit inspiratorischer Druckunterstützung indiziert.

\section{Indikationsstellung der invasiven Atemwegssicherung}

Neben der Reanimationssituation als häufigste Indikation zur prähospitalen Atemwegssicherung sind weitere mögliche Notfallsituationen, bei denen eine invasive Atemwegssicherung erforderlich sein kann:

- die respiratorische Insuffizienz (z. B. bei exazerbierter COPD oder dekompensierter Herzinsuffizienz),

- eine Vigilanzminderung (z. B. Schädel-Hirn-Trauma, intrakranielle Blutung) sowie

- aspirationsgefährdete Patienten (z. B. Intoxikation).

Während bei einer Apnoe oder im Kreislaufstillstand eine sofortige Atemwegssicherung erfolgen muss, liegt bei vorhandener Spontanatmung des Patienten meist eine dringliche Intervention vor, und es besteht noch die zeitliche Reserve für eine Einschätzung der Atemwege und
Vorbereitung der Narkoseinduktion sowie der Präoxygenierung.

Grundsätzlich sollten bei dringlichen Indikationen die eigene Expertise des Teams (Selbsteinschätzung der Erfolgswahrscheinlichkeit der endotrachealen Intubation), logistische Aspekte (z.B. Transportdauer) sowie Begleiterkrankungen und -verletzungen in die Beurteilung mit einbezogen werden. Im Einzelfall kann so z. B. bei kurzer Transportdauer und erwartbar schwierigem Atemwegsmanagement der Patient nach Abwägung unter erhaltener Spontanatmung in den nächstgelegenen, geeigneten Schockraum transportiert werden („Load \& Go“).

Merke

Entscheidend sind die sichere Aufrechterhaltung einer ausreichenden Oxygenierung und Ventilation sowie ein ausreichender Aspirationsschutz und die größtmögliche Sicherheit für den Patienten; diese Punkte sind im Sinne einer Einzelfallentscheidung kritisch abzuwägen.

\section{TAKE HOME MESSAGE}

Die Indikation zur invasiven Atemwegssicherung sollte grundsätzlich streng gestellt werden und dabei die Expertise des Rettungsteams, logistische und patientenbezogene Faktoren in die Überlegung einbezogen werden. Das Unterlassen der invasiven Atemwegssicherung vor Ort kann nach kritischer Abwägung in bestimmten Situationen angezeigt sein.

Bei der Teamentscheidung zur invasiven Atemwegssicherung ist die strukturierte und sorgfältige Vorbereitung essenziell, um mögliche Risiken (z.B. anatomische Besonderheiten, Begleiterkrankungen, Allergien) zu antizipieren und auf Komplikationen (z. B. unerwartet schwieriger Atemweg, hämodynamische Instabilität) bestmöglich vorbereitet zu sein [11]. Auch in zeitkritischen Notfallsituationen sollte die Vorbereitung strukturiert („Checkliste“) erfolgen und analog der Leitlinie „Prähospitale Notfallarkose“ [2] der geplante Ablauf, das Material und die Medikation vorab im Team abgesprochen werden.

\section{TIPP}

Während der Präoxygenierung des Patienten besteht meist ausreichend Zeit, nochmals ein kurzes „TeamTime-out" durchzuführen, die Vollständigkeit der Vorbereitung abzufragen sowie das geplante Vorgehen und die Aufgabenverteilung abzustimmen. 
Die prähospitale Notfallnarkose sollte stets als Rapid Sequence Induction (RSI) durchgeführt werden, da bei Notfallpatienten eine fehlende Nüchternheit und damit Aspirationsgefahr angenommen werden muss [1,2,7].

\section{Beutel-Masken-Beatmung}

Die Beutel-Masken-Beatmung (Bag-Mask-Ventilation, BMV) ist eine grundlegende Maßnahme des prähospitalen Atemwegsmanagements, die von allen Berufsgruppen im Rettungsdienst zu beherrschen ist [1].

Indikation: Die BMV dient

- als Basismaßnahme zur assistierten Beatmung (z. B. bei Bradypnoe oder Apnoe bei Intoxikationen oder Sedierungsüberhang),

- als Erstmaßnahme zur Sicherung der Oxygenierung und Ventilation bei der kardiopulmonalen Reanimation;

- sie ist aber auch als Rückfallebene in allen Notfallsituationen, insbesondere bei einer erschwerten Atemwegssicherung geeignet [1].

\section{Merke}

Indikationen zur BMV sind letztlich die Beatmung vor Durchführung einer endotrachealen Intubation bei bestehender Apnoe, die Rückfallebene bei gescheiterten Intubationsversuchen zur Aufrechterhaltung der Oxygenierung oder die assistierte Beatmung bei kurzzeitiger respiratorischer Insuffizienz (z. B. bei Intoxikationen, Sedierungsüberhang).

In den letzten Jahren hat die BMV im Rettungsdienst durch die Etablierung der extraglottischen Atemwegshilfsmittel (z. B. Larynxtubus, Larynxmaske) bei Reanimationssituationen als primäre Maßnahme an Bedeutung verloren und dient vor allem als überbrückende Maßnahme.

Grundsätzlich sollte jeder Rettungsdienstmitarbeiter die Beutel-Masken-Beatmung beherrschen, und die Fertigkeiten zur Anwendung sollen in der klinischen Ausbildung erlernt werden [1].

Einige Studien haben die BMV bei der Atemwegssicherung in Reanimationsstudien im Vergleich zur invasiven Atemwegssicherung untersucht. Hier zeigt sich ein Trend, dass die BMV bei Patienten unter Reanimation nicht zu schlechteren Behandlungsergebnissen führt $[12,13]$. In den Empfehlungen des International Liaison Committee on Resuscitation (ILCOR-Empfehlungen) zum Atemwegsmanagement für die kardiopulmonale Reanimation [13] wird die BMV ebenfalls berücksichtigt; im Einzelfall kann bei vermuteter geringer Intubationswahrscheinlichkeit eine BMV bis zur Klinik fortgeführt werden. Weiterhin sollte die endotracheale Intubation aber als Goldstandard in Reanimationssituationen angesehen werden. Bei Vorliegen einer „Difficult Airway“-Situation kann auf den extraglottischen Atemweg zurückgegriffen und die BMV als (intermittierende) Rückfallebene genutzt werden.

Durchführung: Bei der Durchführung der BMV sollte bei allen Patienten beachtet werden, dass neben der optimalen Größe und Position der Beatmungsmaske die korrekte Durchführung des C-Griffs zu prüfen ist, Oropharyngealtuben zur Unterstützung einzusetzen sind und bei erschwerter Maskenbeatmung das Verfahren mit zwei Helfern durchzuführen („doppelter C-Griff“) ist. Auch bei der BMV ist die Kapnografie hilfreich, um eine Normokapnie anzustreben [1]. Eine leichte Reklination des Kopfes resp. eine Unterpolsterung des Kopfes („verbesserte JacksonPosition“) erleichtern die Durchführung der Maskenbeatmung.

Die Nutzung eines ausreichenden und situationsangepassten Sauerstoff-Flows sowie eines Reservoirbeutels bzw. eines Demandventils sind obligat [1]. Durch eine prolongierte BMV ist eine Magenüberblähung möglich, sodass im Verlauf nach invasiver Atemwegssicherung eine Entlastung mittels Magensonde erforderlich werden kann. Dies ist insbesondere bei pädiatrischen Patienten klinisch relevant, da die Beatmungssituation durch den erhöhten gastralen Druck erschwert sein kann.

\section{TAKE HOME MESSAGE}

Die Beutel-Masken-Beatmung (BMV) sollte von jedem Rettungsdienstmitarbeiter beherrscht werden. Sie ist als Rückfallebene insbesondere beim schwierigen Atemweg angezeigt.

\section{Invasive Atemwegssicherung}

Die endotracheale Intubation gilt weiterhin als Goldstandard der invasiven Atemwegssicherung in der Notfallmedizin $[1,7]$. Nicht nur der optimale Aspirationsschutz, auch die sichere und effektive Beatmung mit hohen Inspirationsdrücken (z.B. bei COPD, Adipositas) und positivendexspiratorischem Druck (PEEP) sind von Vorteil. Bei Reanimationssituationen führt die kontinuierliche Thoraxkompression und Beatmung zu einer Optimierung des Herzzeitvolumens im Vergleich zu einem asynchronen Vorgehen bei einliegender EGA [9]. Auch Patienten mit einem Schädel-Hirn-Trauma profitieren von einer invasiven Beatmung mittels Endotrachealtubus [14].

Einige Studien belegen den Stellenwert des „First Pass Success“ bei der endotrachealen Intubation [1]. Mit zunehmender Anzahl der Intubationsversuche nehmen Komplikationen wie Hypoxie, Aspiration oder Hypotonie zu. Weitere mit der Atemwegssicherung assoziierten Komplikationen (z. B. Schleimhautschwellungen, Blutun- 
gen, Zahnverletzungen) können nachfolgende Intubationsversuche erschweren. Wiederholte Intubationsversuche unter Reanimationsbedingungen verschlechtern das Behandlungsergebnis [1]. Neben der Expertise durch Ausbildung und Training können weitere Maßnahmen zu einer höheren Erfolgswahrscheinlichkeit des ersten Intubationsversuches beitragen $[15,16]$ :
- eine standardisierte und optimale Vorbereitung inklusive Präoxygenierung,

- ausreichende Narkosetiefe (z.B. noch unzureichende Muskelrelaxierung),

- bestmögliche Lagerung des Patienten,

- Einsatz der Videolaryngoskopie.

\section{FAZIT}

\section{Vorgehen bei der invasiven Atemwegssicherung}

Zum Vorgehen bei der invasiven Atemwegssicherung empfiehlt sich in der rettungsdienstlichen Praxis folgendes Vorgehen

(nach $[1,2]$ ):

- Indikationsstellung zur endotrachealen Intubation kritisch prüfen:

- Bestehen Alternativen?

- Ist ein Transport unter Spontanatmung oder NIV-Therapie möglich bzw. vertretbar?

- Risiken und Besonderheiten (Evaluation des Atemwegs) prüfen.

- Abstimmung des Vorgehens im Rettungsteam:

- Dringlichkeit festlegen: sofortige Intubation versus dringliche Intubation.

- Ort der Maßnahmen (Wohnung/Einsatzstelle versus RTW) festlegen.

- Material, Medikation und Zuständigkeiten abstimmen:

- Tubusgröße (inkl. alternativer Größen),

- Spatelgröße,

- Dosierung der Induktionsmedikamente,

- Aufgabenverteilung im Team (Assistenz bei der Intubation usw.).

- Optimale Lagerung des Patienten (Oberkörperhochlagerung, verbesserte Jackson-Position, ggf. Zahnprothesen entfernen).

- Präoxygenierung (Sauerstoffinsufflation, CPAP/NIV-Therapie), ggf. assistierte Beatmung, falls erforderlich $\left(\mathrm{FiO}_{2} 1,0\right.$, Reservoirbeutel, ggf. Demandventil).

- Anlage von 1-2 gut laufenden peripheren Verweilkanülen, ggf. Nutzung eines liegenden Portsystems oder einer intraossären Kanüle.

- Komplettierung des Monitorings (EKG, NIBP [nicht am Infusionsarm] z. B. 1-minütlich, $\mathrm{SpO}_{2}$, etCO $\mathrm{CO}_{2}$ ).

- Sorgfältige und standardisierte Vorbereitung des Materials zur Atemwegssicherung, inklusive Material für den unerwartet schwierigen Atemweg (z. B. EGA, Koniotomieset), Absaugung, Führungsstab, Kapnografie sowie komplette Medikation (z. B. Induktionsmedikamente, Katecholamine).

- Ansage der Induktionsmedikamente und Abwarten der Anschlagszeit der Muskelrelaxierung (sofern möglich, keine Zwischenbeatmung bei Rapid Sequence Induction, Ausnahme: pädiatrische Patienten).
- Laryngoskopie (maximal 30 s, dann Abbruch des Intubationsversuchs), ggf. BURP-Manöver.

- Bei primär erfolgreicher Intubation:

- Blockung des Endotrachealtubus, Lagekontrolle und obligate Kapnografie, Tubusfixierung, Cuffdruckmessung, Dokumentation.

- Bei erfolgloser Intubation:

- Zwischenbeatmung (Beutel-Maske, $\mathrm{FiO}_{2} 1,0$ ).

- Optimierung vor zweitem Intubationsversuch: Lagerung, Narkosevertiefung, Equipment (Spatel u. a.), ggf. BURPManöver.

- Erneuter, maximal zweiter Intubationsversuch.

- Bei erneuter erfolgloser Intubation:

- Maximal 2 Versuche zur Anlage eines EGA (Larynxtubus, -maske).

- Bei Erfolglosigkeit:

- Entscheidung: Transport unter (suffizienter) BMV,

- oder bei „Cannot intubate, cannot oxygenate“ (CICO): Koniotomie.

- Lagekontrolle: Auskultation, Kapnografie, Pulsoxymetrie.

Dokumentation.

- Sichere Intubationszeichen:

- Intubation unter Sicht?

- Kapnografie positiv?

- (Unsicher: seitengleiche Thoraxexkursionen bei manueller Beatmung.)

- Reevaluation (nach ABCDE): Oxygenierung, Normokapnie, Tubuslage (Tiefe in Höhe der Zahnreihe mit Dokumentation).

- Beurteilung der Narkosetiefe:

- hämodynamische Stabilität nach Narkoseinduktion,

- ggf. Anlage einer Magensonde.

- Beatmungsmanagement:

- Beatmungsmodus: volumen- versus druckkontrolliert.

- $\mathrm{FiO}_{2}$ (Anpassung nach Situation, z. B. nach CPR).

- PEEP (nach Oxygenierung/analog zum $\mathrm{FiO}_{2}$ ).

- Normokapnie.

- Repetitive Gaben von Narkotika, ggf. weitere Relaxierung. 


\section{Merke}

Der „First Pass Success“, also der Intubationserfolg im ersten Intubationsversuch, ist prognostisch bedeutsam, daher soll die Atemwegssicherung auch prähospital unter bestmöglichen Bedingungen durchgeführt werden.

Auch wenn prospektive Studien zum aktuellen Zeitpunkt fehlen, so ist mittlerweile der primäre Einsatz der Videolaryngoskopie in Notfallsituationen prähospital etabliert. Eine verbesserte Sicht auf die Stimmbandebene, eine verminderte Reklination der Halswirbelsäule insbesondere beim Trauma sind belegte Vorteile [14,15]. Primär sollen auch bei der Videolaryngoskopie Macintosh-ähnliche Spatel eingesetzt werden, um im Falle von Equipmentversagen unmittelbar von der indirekten auf die direkte Laryngoskopie wechseln zu können. Anatomisch geformte Spatel sollten erfahrenen Nutzern und speziellen Notfallsituationen vorbehalten sein.

Die Anwendung eines Führungsstabes (z. B. EschmannStab) kann unterstützend hilfreich sein, bei absehbar schwieriger Intubation sollte er primär verwendet werden. In jedem Fall muss die Handhabung des EschmannStabes jedem im Team bekannt sein und regelmäßig beübt werden.

\section{TIPP}

Die Videolaryngoskopie sollte primär bei jeder Atemwegssicherung erfolgen, um einen möglichst hohen Intubationserfolg („First Pass Success“) zu erzielen.

\section{Der schwierige Atemweg in der prähospitalen Notfallmedizin}

Unter Notfallbedingungen ist prähospital als auch innerklinisch immer mit einer erschwerten Atemwegssicherung zu rechnen. Situationsbezogene (z. B. räumliche Enge am Einsatzort, Dunkelheit, eingeklemmter Patient im Fahrzeug) und patientenbezogene Faktoren (z. B. fehlende Nüchternheit, ggf. fehlende Möglichkeit der Anamnese) erschweren die Durchführung der Atemwegssicherung. Hier liegt dann eine grundsätzlich andere Situation vor als etwa bei einer elektiven Narkoseeinleitung und Atemwegssicherung z.B. in der Anästhesie. Hinzu kommen wechselnde Rettungsteams und die unterschiedliche Expertise des notärztlichen und rettungsdienstlichen Personals.

\section{FALLBEISPIEL}

\section{Fortsetzung}

Im RTW wird ein erneutes Team-Time-out durchgeführt, die Vollständigkeit der Vorbereitung des Materials und der Medikation vom Teamleiter abgefragt, die Aufgaben im Team werden verteilt (Intubationsassistenz, Injektion der Medikation, manuelle Inline-Stabilisation der HWS) und nach positiver Rückmeldung die Narkoseinduktion begonnen (0,2 mg Fentanyl, $150 \mathrm{mg}$ Propofol 1\%, $100 \mathrm{mg}$ Rocuronium unter frei laufender Kristalloidgabe). Währenddessen wird die Präoxygenierung fortgeführt und das Monitoring inklusive 1-minütlicher nichtinvasiver Blutdruckmessung und akustischem $\mathrm{SpO}_{2}$-Signal vorbereitet.

Unter stabiler Oxygenierung $\left(\mathrm{SpO}_{2}\right.$ stets $\left.>95 \%\right)$ wird die Anschlagszeit des Muskelrelaxans abgewartet und unter manueller Inline-Stabilisierung (MILS) die Videolaryngoskopie durchgeführt. Hierbei ist die Stimmbandebene nicht sofort darstellbar. Mittels eines BURP-(Backward-Upright-Rightward-Pressure-)Manövers gelingt die Einstellung, allerdings ist der Endotrachealtubus nicht unmittelbar einzuführen. Nach rund 30 Sekunden und bei beginnendem Abfall der Sauerstoffsättigung < $95 \%$ wird der erste Intubationsversuch unterbrochen, unter fortgeführter manueller InlineStabilisierung die Beutel-Masken-Beatmung fortgeführt und der Patient zunächst manuell beatmet, bis eine ausreichende Oxygenierung eintritt.

In einem erneuten Team-Time-out wird evaluiert, wie der zweite Intubationsversuch optimiert werden kann: Lagerung, Narkosetiefe und Spatel- und Tubusgröße werden als ausreichend beurteilt. Bei guter videolaryngoskopischer Sicht auf die Stimmbandebene wird ein Eschmann-Stab vorbereitet, zudem werden Larynxtuben der Größe 4 und 5 als Alternative berücksichtigt. Bei adäquater Oxygenierung unter problemloser Maskenbeatmung ohne Anzeichen einer Regurgitation wird der zweite Intubationsversuch durchgeführt, dieser gelingt mithilfe des Eschmann-Stabes problemlos.

\begin{abstract}
Merke
In allen Notfallsituationen muss mit einem unerwartet schwierigen Atemweg gerechnet werden, Komplikationen und Nebenwirkungen z. B. der Narkoseinduktion müssen antizipiert werden, und es muss eine entsprechende sorgfältige und standardisierte Vorbereitung erfolgen $[1,7,11]$.
\end{abstract}

Essenziell zur Bewältigung der insgesamt seltenen Situation des schwierigen Atemwegs ist, dass alle Mitglieder des Rettungsteams und insbesondere der Notarzt als Teamleiter einen Notfallalgorithmus verinnerlicht haben, der in belastenden Notfallsituationen wie einer "Cannot intubate, cannot oxygenate“-Situation (CICO) strukturiert und Schritt für Schritt abgearbeitet werden kann. Die S1-Leitlinie „Prähospitales Atemwegsmanagement“ bietet hierzu einen Universalalgorithmus, der in jedem Rettungsdienstbereich unter Berücksichtigung lokaler 
Gegebenheiten und der Vorhaltung entsprechenden Materials angewandt werden kann (s. - Abb. 1) und Sicherheit und Struktur in der Notfallsituation bietet.

\section{Erwartet schwieriger Atemweg}

Beim erwartet schwierigen Atemweg aufgrund anatomischer Besonderheiten oder anamnestischer Hinweise des Patienten oder der Angehörigen bzw. Vorliegen eines Anästhesieausweises sollte prähospital die Intubationsindikation sehr streng gestellt werden, da prähospital nicht alle in der Klinik vorgehaltenen Optionen (z. B. fiberoptische Intubation) zur Verfügung stehen. Ein vielerorts sich etablierendes Oberarzt-Rettungsdienst-System wäre bei Vorliegen eines solchen erwarteten schwierigen Atemwegs frühzeitig hinzuzuziehen.

In jedem Fall aber ist ein Algorithmus einzuhalten, der aktuell nach maximal 2 Intubationsversuchen und 2 Versuchen zur Etablierung eines extraglottischen Atemwegs die Möglichkeit der BMV zur suffizienten Oxygenierung bis in die Klinik sieht. Diese Einzelfallentscheidung ist vom Notarzt bei der Übergabe zu begründen, vom klinischen Personal zu akzeptieren und nicht als prähospitales Versorgungsdefizit, sondern im Sinne der Patientensicherheit zu sehen.

\section{TAKE HOME MESSAGE}

Ein erwartet schwieriger Atemweg sollte im Vorfeld evaluiert und das Atemwegsmanagement ggf. modifiziert werden. Im Einzelfall ist nach Abwägung bei ausreichender Oxygenierung eine Atemwegssicherung erst unter klinischen Bedingungen (z. B. im Schockraum) angezeigt und sicherer für den Patienten.

\section{Präoxygenierung}

Durch eine sorgfältige Präoxygenierung besteht im Idealfall ausreichend zeitliche Reserve, den Algorithmus im Rettungsteam abzuarbeiten, die Beutel-Masken-Beatmung als Rückfallebene zu nutzen und alternative Methoden der Atemwegssicherung anzuwenden ( $\triangleright$ Abb. 1).

Zur Präoxygenierung kann die Sauerstoffgabe beim spontanatmenden Patienten via Inhalationsmaske $(15 \mathrm{l} / \mathrm{min}$, Reservoirbeutel), im Rahmen einer CPAP/NIV-Therapie erfolgen oder aber über eine dicht sitzende Beatmungsmaske eines Beatmungsbeutels $\left(\mathrm{FiO}_{2}\right.$ 1,0, Reservoirbeutel, ggf. Demandventil). Die Präoxygenierung sollte im Idealfall etwa 3-4 Minuten durchgeführt werden, zumindest sollte eine ausreichend periphere Sauerstoffsättigung $\left(\mathrm{SpO}_{2}>95 \%\right)$ bestehen. Durch ein „Auswaschen“ von Stickstoff (Denitrogenisierung) und Anfluten von Sauerstoff wird die funktionelle Residualkapazität bestmöglich genutzt, um eine potenzielle Apnoetoleranz im Rahmen des Atemwegsmanagements zu verlängern.

\begin{abstract}
Merke
Eine Präoxygenierung sollte daher bei jeder Atemwegssicherung durchgeführt werden $[1,7]$.
\end{abstract}

Ein weiteres Verfahren zur apnoeischen Oxygenierung ist die vorherige Anlage einer Sauerstoffnasenbrille unter der Inhalations-, NIV- oder Beatmungsmaske, sodass während der Laryngoskopie weiterhin eine Sauerstoffzufuhr besteht und damit zusätzlich eine Desaturierung verlangsamt werden kann [1].

\section{TAKE HOME MESSAGE}

Die Präoxygenierung ist bei jeder Atemwegssicherung obligat, um die Zeitdauer zwischen Beginn der Apnoe und dem Abfall der $\mathrm{SpO}_{2}$ zu verlängern. Ein unerwartet schwieriger Atemweg muss bei jeder prähospitalen Atemwegssicherung bedacht werden. Ein standardisierter Algorithmus zur Problemlösung muss strukturiert abgearbeitet werden.

\section{Komplikationen und Notfallmanagement}

Da die prähospitale Atemwegssicherung unter suboptimalen Bedingungen, in wechselnden Teams stattfindet und bei vitaler Bedrohung des Patienten oft zeitkritisch ist, sind zahlreiche Komplikationen möglich $[16,17]$, aber insgesamt erwartbar und meist durch optimale Vorbereitung, Antizipation sowie Ausbildung und Training beherrschbar. Mögliche Komplikationen sind in der Übersicht zusammengefasst.

All diese Komplikationen sollten in der Notfallsituation antizipiert werden, sie können durch eine strukturierte und sorgfältige Vorbereitung zum Teil vermieden werden. Jedoch sollte das gesamte Team auf die Beherrschung der Komplikationen vorbereitet sein. Ein strukturiertes Vorgehen und Reevaluation (ABCDE) hilft, Ursachen rasch und systematisch zu detektieren.

Die erschwerte Laryngoskopie wird prähospital deutlich häufiger beschrieben als innerklinisch [1]. Die Rate an Tubusfehllagen ist erhöht $[16,17]$, und es sind zahlreiche patienten- und einsatzseitige Faktoren beschrieben, die die prähospitale Intubation erschweren:

- patientenseitige Faktoren: z. B. Verletzungen der oberen Atemwege, Verlegung durch Blut u.a., eingeschränkte Immobilisation der Halswirbelsäule beim Trauma,

- einsatzseitige Faktoren: Intubation unter CPR, limitierter Zugang zum Patienten bei Traumata, Umgebungsverhältnisse usw. 


\section{ÜBERSICHT}

Mögliche Komplikationen bei der Atemwegssicherung

- Hypoxie bei insuffizienter Maskenbeatmung („Cannot ventilate“) und frustraner Laryngoskopie („Cannot intubate“) mit möglicher konsekutiver Bradykardie und Asystolie

- Hypotension (Hypovolämie, Trauma), insbesondere verstärkt durch die Narkoseinduktion

- anaphylaktische Reaktionen nach Gabe der Induktionsmedikamente

- Magenüberblähung (insbesondere bei prolongierter Maskenbeatmung)

- Regurgitation und Aspiration

- Zahnverletzungen

- Blutungen im Mund-/Nasen-/Rachenraum (bei wiederholten Laryngoskopieversuchen)

- Schleimhautschwellungen und Ödembildung

- Laryngospasmus

- Tubusfehllagen (einseitige Lage rechtsbronchial, ösophageale Fehllage)

- Hypo- oder Hyperkapnie bei fehlendem Monitoring (Kapnografie) bzw. bei manueller Beatmung

- Pneumothorax bzw. Spannungspneumothorax insbesondere nach erfolgter Intubation (Ruptur von Bullae, vorbestehender Pneumothorax z. B. bei Thoraxtrauma)

- die Möglichkeit von technischem Versagen des Equipments zur Atemwegssicherung

Sofern zeitlich und situativ möglich, sollten diese Faktoren vorab evaluiert und im Atemwegsmanagement berücksichtigt werden.

\section{TAKE HOME MESSAGE}

Komplikationen der invasiven Atemwegssicherung müssen vom Rettungsteam rasch erkannt und behandelt werden. Ein strukturiertes Vorgehen kann häufige Komplikationen wie Hypoxie, Hypotension oder Tubusfehllagen meist detektieren.

\section{Stellenwert der extraglottischen Atemwegssicherung im Rettungsdienst}

In den letzten Jahren hat der extraglottische Atemweg (EGA) einen zunehmend wichtigen Stellenwert in der prähospitalen Notfallmedizin erhalten. Sowohl der primäre Einsatz (z.B. bei Reanimationssituationen) durch Rettungsdienstfachpersonal oder die alternative Verwen- dung bei erfolgloser Laryngoskopie sind etablierte Verfahren.

Die Ausbildung im Umgang mit EGA ist teilweise unter Elektivbedingungen möglich. Die empfohlenen Fallzahlen zum Erlernen sind niedriger als bei der endotrachealen Intubation. Die Erfolgsrate ist bei korrekter Anwendung hoch, und die Komplikationsrate ist unter Beachtung wichtiger Kontraindikationen und Herstellerangaben geringer als bei der endotrachealen Intubation. Allerdings wurden in den letzten Jahren auch Komplikationen bei der Anwendung von EGA beschrieben, und Sicherheitsvorgaben wie z.B. die obligate Cuffdruckmessung wurden angepasst. So sind SOP/SAA für Rettungsdienstfachpersonal zum Einsatz der EGA vorgegeben [4,5]. Weitere Empfehlungen sehen auch bei EGA eine Mindestzahl von 45 Anwendungen am Patienten vor, zudem sind regelmäßige Fortbildungen zum Umgang mit EGA in Rettungsdienstbereichen sinnvoll.

Eine weitere Empfehlung sieht die Cuffdruckmessung

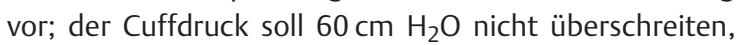
um Komplikationen wie Schleimhautläsionen, Blutungen oder Nervenverletzungen zu minimieren.

Grundsätzlich sollten EGA der 2. Generation verwendet werden, die auch die Möglichkeit einer gastralen Absaugung bzw. Entlastung enthalten. Zudem ist bei der Anwendung von EGA auf eine ausreichende Dichtigkeit zu achten (Nebengeräusche). Die Anwendung der Kapnografie ist obligat. Bei insuffizienter Oxygenierung oder Ventilation muss ein alternatives Verfahren gewählt werden (BMV oder Notfallkoniotomie als Ultima Ratio) [1].

\section{Notfallkoniotomie}

Durch den Einsatz der Videolaryngoskopie, aber auch der EGA sowie strukturierter Handlungsalgorithmen hat der Stellenwert der Notfallkoniotomie prähospital abgenommen. Nichtsdestotrotz stellt die Notfallkoniotomie in manchen lebensbedrohlichen Situationen eine Ultima Ratio bei einer "Cannot Intubate, Cannot Oxygenate“ (CICO)-Situation dar und ist fester Bestandteil gängiger Atemwegsalgorithmen $[1,7,11,14]$.

Indikation: Die Notfallkontiotomie muss theoretisch und praktisch von ärztlichem Rettungsdienstfachpersonal beherrscht und bei erfolgloser Atemwegssicherung ohne Zeitverzug durchgeführt werden, bevor es zu einer lebensbedrohlichen Hypoxie kommt [1]. Eine erfolglose Atemwegssicherung ist gekennzeichnet durch

- maximal 2 Intubationsversuche <30 s Dauer,

- maximal 2 Versuche der Etablierung eines extraglottischen Atemwegs (EGA),

- insuffiziente Beutel-Masken-Beatmung. 
Praktische Übungen sind am Leichen- oder Tierpräparat möglich.

Grundsätzlich sollte bei Kindern eine besonders strenge Indikation zur Koniotomie gestellt werden, da die Komplikationsrate deutlich erhöht ist und durch weniger invasive Maßnahmen wie einen „Rachentubus“ oder die BMV häufig eine suffiziente Oxygenierung erreicht werden kann [1].

Durchführung: Verschiedene technische Möglichkeiten bestehen zur Durchführung einer Notfallkoniotomie, die Vorhaltung des Materials variiert in den verschiedenen Rettungsdienstbereichen.

- Eine direkte Punktionstechnik mittels Kanüle („Catheter-over-Needle Technique“, z.B. QuickTrach) ist weit verbreitet.

- Alternativ kann mittels Seldinger-Technik das Lig. cricothyroideum punktiert und nach Dilatation eine Trachealkanüle eingeführt werden (z.B. Technik nach Melker).

- Universell einsetzbar, da mit üblichem Notfallmaterial durchführbar, ist die chirurgische Notfallkoniotomie, bei der mit einem Skalpell das Lig. cricothyroideum durchtrennt und dann ein dünner Endotrachealtubus (z. B. ID 6,0 oder $6,5 \mathrm{~mm}$ ) oder eine Trachealkanüle eingeführt wird, ggf. nach Dilatation mithilfe eines Spekulums.

- Im angelsächsischen Sprachraum ist das „FONA-Schema“ (Front-of-Neck Access) etabliert, bei dem mit einem Skalpell Haut und Lig. cricothyroideum durchtrennt werden. Ein weicher Führungsstab (EschmannStab) wird eingelegt, über den dann ein Endotrachealtubus eingeführt wird [18].

\section{Cave}

Mögliche Komplikationen bei der Notfallkoniotomie sind

- Verletzungen der Tracheahinterwand (insbesondere bei der direkten Punktion),

- Bildung eines Hautemphysems und

- Blutungskomplikationen (bei allen Verfahren).

Jedes der genannten Verfahren zeigt Vor- und Nachteile in der Anwendung, wobei insbesondere das chirurgische Verfahren technisch einfach durchführbar ist und kein spezielles Material vorgehalten werden muss. Jeder Rettungsdienstmitarbeiter sollte mit dem vorgehaltenen Material vertraut sein, und die Notfallkoniotomie sollte regelmäßiger Bestandteil von Fortbildungen zum Atemwegsmanagement sein [1].
TIPP

Die apnoeische Oxygenierung (z. B. über einen Eschmann-Stab) stellt in Ausnahmesituationen eine weitere Alternative dar, um eine minimale Oxygenierung zu ermöglichen.

\section{TAKE HOME MESSAGE}

Die Notfallkoniotomie ist als Ultima Ratio fester Bestandteil aller „Difficult Airway“-Algorithmen und muss von jedem Notarzt beherrscht werden.

\section{FALLBEISPIEL}

\section{Fortsetzung}

Nach erfolgreicher atraumatischer Intubation unter videolaryngoskopischer Sicht erfolgt die strukturierte Lagekontrolle. Die Kapnografie zeigt einen adäquaten et $\mathrm{CO}_{2}(42 \mathrm{mmHg})$, bei der Auskultation wird ein fehlendes Geräusch epigastrisch und ein seitengleiches vesikuläres Atemgeräusch festgestellt. Die Tubustiefe wird mit $20 \mathrm{~cm}$ auf Höhe der Zahnreihe dokumentiert, und der Endotrachealtubus wird fixiert. Schließlich wird die HWS-Orthese erneut befestigt.

Anschließend wird unter fortlaufendem Monitoring bei weiterhin stabiler Hämodynamik die kontrollierte Beatmung am Transportbeatmungsgerät eingestellt (PCV, Pinsp 20 mbar, AF 14/min, $\mathrm{FiO}_{2}$ 0,8, PEEP 7 mbar). Als Zielparameter werden eine $\mathrm{SpO}_{2}$ von $>95 \%$ sowie ein et $\mathrm{CO}_{2}$ von $35-40 \mathrm{mmHg}$ für den Transport definiert. Vor Transportbeginn erfolgt eine Reevaluation im Sinne des ABCDE, zudem wird die Narkose mittels Midazolam-Bolusgaben aufrechterhalten und eine Magensonde gelegt.

Der Transport erfolgt nach telefonischer Voranmeldung in den Schockraum der nächsten geeigneten Klinik. 


\section{Beatmungsmanagement nach erfolgreicher Atemwegssicherung}

Nach erfolgter Atemwegssicherung (ETI oder EGA) und Lagekontrolle wird der Notfallpatient in der Regel kontrolliert beatmet. Ziele sind hierbei

- eine alters- und gewichtsadaptierte Beatmungstherapie (z.B. 5-7 ml/kgKG Tidalvolumen) und

- eine Normoxie $\left(\mathrm{SpO}_{2}>95 \%\right)$ und

- Normokapnie (etCO $25-45$ mmHg).

Bei einer Vielzahl an Transportbeatmungsgeräten und Beatmungsmodi verzichten wir hier auf konkrete Angaben zu Beatmungsparametern. Obligat ist neben einem kompletten Monitoring inklusive der Kapnografie (100\% Kapnografierate) auch die Fortführung der Notfallnarkose. Parameter wie die Herzfrequenz und der nichtinvasive Blutdruck, Spontanatmung des Patienten, Schwitzen etc. können Hinweis auf die Narkosetiefe geben.

Nach jeder Umlagerung oder Übergabe sollte nach dem ABCDE-Schema der Patientenzustand evaluiert werden und z. B. zum Ausschluss einer Tubusdislokation eine Auskultation erfolgen. Eine manuelle Beatmung sollte nur in Ausnahmefällen (z. B. bei kurzer Transportzeit) durchgeführt werden, da u. a. die Gefahr einer Hypo- bzw. Hyperventilation besteht.

Treten nach erfolgter Atemwegssicherung unter kontrollierter Beatmung Probleme wie ein Anstieg des Beatmungsdrucks, ein Blutdruck- oder Sättigungsabfall auf, sollte eine sofortige Reevaluation erfolgen (ABCDE). Eine systematische Fehleranalyse kann mithilfe des „DOPES“Akronyms erfolgen ( $\triangleright$ Tab. 1), mit dem die häufigsten Ursachen erfasst und systematisch abgearbeitet werden können.

\section{TAKE HOME MESSAGE}

Ziele der Beatmungstherapie sind eine Normoxie, Normokapnie sowie eine ausreichende Sedierungstiefe.

Eine standardisierte Reevaluation sollte nach Umlagerungen, Übergaben oder bei Änderungen des Patientenzustands erfolgen.

\section{Atemwegssicherung bei pädiatrischen Patienten}

Bei pädiatrischen Patienten gibt die S1-Leitlinie „Prähospitales Atemwegsmanagement“ explizite Empfehlungen, die von den Vorgaben für Erwachsene abweichen. Abhängig von der Erfahrung des Notarztes im Umgang mit pädiatrischen Patienten sollte die Wahl der Atemwegssicherung erfolgen:

So ist bei häufig geringerer Expertise vieler Notärzte bei der endotrachealen Intubation im Kindesalter eine ausreichende Oxygenierung und Ventilation auch mittels Beutel-Masken-Beatmung möglich. Die optimierte Maskenbeatmung bei Kindern beinhaltet

- eine optimale Kopflagerung,

- eine korrekte Maskengröße,

- ggf. den Einsatz eines passenden Oropharyngealtubus sowie

- den Einsatz des „doppelten C-Griffs“.

Diese optimierte assistierte bzw. kontrollierte BeutelMasken-Beatmung ist technisch meist einfach durchzuführen. Das Verfahren kann bei pädiatrischen Notfällen sowohl als primäres Verfahren angewandt als auch als Rückfallebene bei erschwerter Atemwegssicherung genutzt werden („Plan A“).

- Tab. 1 Differenzialdiagnosen von „A-“ und „B-Problemen“ nach Atemwegssicherung - „DOPES“-Schema [1].

\begin{tabular}{|l|l|l|}
\hline Akronym & Erklärung & $\begin{array}{l}\text { akzidentelle Extubation, einseitige Intubation, ösophageale oder } \\
\text { hypopharyngeale Fehllage }\end{array}$ \\
\hline D & Dislokation des Tubus & Trachealtubus, HME-Filter, Beatmungsschläuche \\
\hline O & Obstruktion & Pneumonie, Bronchospastik, Lungenödem, Pleuraerguss, Fremdkörper \\
\hline P & Pneumothorax und andere pulmonale Störungen & Sauerstoffquelle, Beatmungsbeutel, Beatmungsleckage \\
\hline E & Equipmentversagen & $\begin{array}{l}\text { Magenüberblähung nach Beutel-Masken-Beatmung; Herzinsuffizienz, } \\
\text { Shunt }\end{array}$ \\
\hline S & Stomach = Magenüberblähung und Spezielles & \\
\hline
\end{tabular}

$\mathrm{HME}=$ Heat and Moisture Exchanger 
Als alternatives Verfahren kann im Notfall eine supraglottische Atemwegssicherung erfolgen („Plan B“). Hierbei wird aufgrund der aktuellen Datenlage eindeutig der Einsatz der Larynxmaske, idealerweise der 2. Generation empfohlen [1]. Die Larynxmaske ist auch im Neugeborenenalter einsetzbar, und es besteht ausreichende klinische Erfahrung bei der Anwendung bei pädiatrischen Patienten.

Die Indikation zur endotrachealen Intubation („Plan C“) im Kindesalter sollte aufgrund der Komplikationsrate entsprechend streng gestellt werden und nur bei vital bedrohten Patienten und entsprechend situationsbezogenen Faktoren (eher längere Transportzeiten und nur bei entsprechender Expertise des Notarztes) erfolgen. Sollte ein Kindernotarzt- oder ein Oberarzt-Rettungsdienst-System vorhanden sein, ist dieser frühzeitig nachzualarmieren.

\section{TAKE HOME MESSAGE}

Normoxie und Normokapnie sind die wesentlichen Therapieziele, die meist auch mittels einer optimierten Maskenbeatmung im Kindesalter erreichbar sind. Die Indikation zur endotrachealen Intubation beim Kind sollte prähospital streng gestellt werden und hängt wesentlich von der Expertise des Notarztes ab.

\section{Atemwegssicherung bei der Reanimation}

In Reanimationssituationen erfolgt initial meist eine Atemwegssicherung durch nichtärztliches Rettungsdienstfachpersonal. Neben der BMV ist im Rettungsdienst der Larynxtubus als häufiges EGA-Device verbreitet, da innerhalb kurzer Zeit eine Atemwegssicherung erfolgen kann. SOP/SAA, insbesondere für Notfallsanitäter, sehen hier Standardalgorithmen vor [4,5]. Im Jahresbericht 2019 des Deutschen Reanimationsregisters ist eine Zunahme der supraglottischen Atemwegssicherung zu verzeichnen [19].

Komplikationen: Auch bei EGA sind allerdings mögliche Komplikationen zu bedenken und müssen bei der Anwendung erkannt und antizipiert werden [16], z. B.

- Leckage,

- Dislokationen,

- insuffiziente Ventilation mit Hyperkapnie,

- Blutungen,

- Ödembildung mit relevanter Zungenschwellung,

- Nervenkompression,

- Regurgitation und

- Aspiration.
Dem steht die einfache Handhabung insbesondere im Vergleich mit der BMV gegenüber.

Der Goldstandard bei der Atemwegssicherung im Rahmen der prähospitalen Reanimation (OHCA) stellt weiterhin die endotracheale Intubation dar [9]. Neben einer suffizienten Oxygenierung und Ventilation ist das Aspirationsrisiko deutlich minimiert, zudem bestehen bei kontinuierlicher Kardiokompression und Ventilation nach invasiver Atemwegssicherung auch positive hämodynamische Effekte (verminderte No-Flow Time, erhöhtes Herzzeitvolumen). Der Einsatz der endotrachealen Intubation durch erfahrene Anwender in Reanimationssituationen kann das neurologische Outcome sowie die Überlebensrate der Patienten verbessern [20].

Pragmatisch muss in der jeweiligen Reanimationssituation nach Eintreffen des Notarztes entschieden werden, ob die initial durchgeführte Atemwegssicherung suffizient ist oder eine sekundäre endotracheale Intubation erforderlich ist. Hilfreich sind hierbei die Beurteilung von Thoraxexkursionen, Nebengeräuschen und der Kapnografie. Auch die Fortführung der Beatmung mittels EGA bis zur Übernahme in der Klinik ist insbesondere bei kurzen Transportzeiten vertretbar, um weitere Verzögerungen zu vermeiden. Allerdings müssen hier unbedingt rettungstechnische Aspekte beachtet werden (etwa Rettung über Drehleiter, Transport im Tragetuch durch ein enges Treppenhaus, Einsatz eines ACCD). Auch auf die Dichtigkeit der EGA sowie eine Normoxie und Normokapnie muss geachtet werden; ggf. ist ein Verfahrenswechsel hin zu einer endotrachealen Intubation einzuleiten.

\footnotetext{
Merke

Essenziell ist in allen Situationen, nach dem gängigen ABCDE-Schema insbesondere nach Übernahme, vor und nach dem Transport oder bei klinischer Verschlechterung, den Atemweg zu überprüfen und ggf. zu korrigieren, da eine suffiziente Oxygenierung und Ventilation relevant für das Behandlungsergebnis sind.
}

Im Jahr 2019 wurden aktualisierte ILCOR-Empfehlungen zur Atemwegssicherung bei der Reanimation veröffentlicht [13]. Diese beruhen im Wesentlichen auf 3 Studien zur Atemwegssicherung bei Herz-Kreislauf-Stillstand [21-23] und stellen die Erfolgswahrscheinlichkeit der endotrachealen Intubation in den Vordergrund.

Die ILCOR-Empfehlungen geben dabei keinem Verfahren der Atemwegssicherung unter Reanimation einen klaren Vorzug. Bei erwartbar niedriger Erfolgsrate einer Intubation sollte primär eine Atemwegssicherung mittels EGA erfolgen (schwache Empfehlung, niedrige Sicherheit der Evidenz). Diese Empfehlungen sind jedoch mit niedriger bis moderater Evidenz bewertet, zudem ist die Übertrag- 
barkeit zwischen den verschiedenen Systemen (Paramedic versus arztbesetzte Rettungsmittel) eingeschränkt.

Für die Einsatzpraxis kann allerdings gefolgert werden, dass vor allem im Rahmen eines sogenannten „Erstangriffs “ - also der Beatmung innerhalb der ersten Minuten einer professionellen Versorgung im Rahmen eines Herz-Kreislauf-Stillstands - die Nutzung von EGA gerade durch Rettungsdienstfachpersonal Vorteile bietet. EGA ist auch unter schwierigen Umgebungsbedingungen möglich, hat höhere Erfolgsraten und ist leichter zu erlernen als die endotracheale Intubation und die Maskenbeatmung. Auf diese Weise ist der Realität im deutschen Rettungsdienst Rechnung getragen. Die Beutel-MaskenBeatmung kann ebenfalls als Rückfallebene betrachtet werden, falls eine anderweitige Atemwegssicherung erfolglos ist.

Weitere Studien zur prähospitalen Atemwegssicherung sind zu fordern, insbesondere unter Berücksichtigung der zunehmenden Professionalisierung des nichtärztlichen Rettungsdienstpersonals und des Einsatzes von Verfahren wie der Videolaryngoskopie [1].

\section{TAKE HOME MESSAGE}

Die endotracheale Intubation gilt weiterhin als Goldstandard der Atemwegssicherung bei der Reanimation, die Atemwegssicherung sollte aber abhängig von der Expertise im Rettungsteam durchgeführt werden.

Eine initiale extraglottische Atemwegssicherung ist üblich und kann bei suffizienter Oxygenierung und Ventilation zunächst fortgeführt werden.

\section{Atemwegssicherung beim Traumapatienten}

Auch beim polytraumatisierten Patienten oder anderen Verletzungsmustern gelten die Grundsätze des Primary Survey der Traumaversorgung nach dem (C)ABCDE-Schema. Sichere Atemwege und eine ausreichende Oxygenierung sind hier vorrangig und meist durch Basismaßnahmen (z.B. mechanisches Freimachen der Atemwege, Sauerstoffinsufflation) zu erreichen.

\section{Merke}

Insbesondere beim Traumapatienten ist bei „B-Problemen“ und klinischem Verdacht auf einen Spannungspneumothorax die sofortige Entlastungspunktion bzw. Minithorakotomie und Drainagenanlage indiziert [14].
Indikation: Die Indikation zur endotrachealen Intubation besteht bei Traumapatienten bei insuffizienter Oxygenierung beim Thoraxtrauma $\left(\mathrm{SpO}_{2}<90 \%\right)$ trotz Sauerstoffapplikation sowie bei Vigilanzminderung (z. B. V.a. Schädel-Hirn-Trauma („D-Problem“, GCS $\leq 9$ Punkte) [14].

Während der Patient mit Schädel-Hirn-Trauma von einer invasiven Beatmung (Normoxie, Normokapnie, Aspirationsschutz) hinsichtlich des Behandlungsergebnisses profitiert [14], sollte bei anderen Verletzungsmustern auch hier situations- und anwenderbezogen entschieden werden, ob prähospital eine Atemwegssicherung erfolgen muss oder, z. B. bei kurzen Transportzeiten, eine ausreichende Oxygenierung und Ventilation auch mit Basismaßnahmen wie der Sauerstoffinsufflation oder der BMV erreicht werden kann. Insbesondere beim bewusstseinsgetrübten Patienten mit nicht gesicherten Atemwegen müssen dann die Versorgung und der Transport in sofortiger Absaug- und Intubationsbereitschaft erfolgen.

Ausbildungsformate zur prähospitalen Traumaversorgung favorisieren kurze Versorgungszeiten vor Ort, sodass im Einzelfall eine invasive Atemwegssicherung nach Abwägung unterbleiben bzw. in den Schockraum verlagert werden kann, wenn eine ausreichende Oxygenierung und Ventilation anderweitig erzielt wird [24]. Entscheidend ist dann die adäquate Voranmeldung im Schockraum, damit entsprechende Vorbereitungen getroffen werden können.

\section{Atemwegsmanagement beim tracheotomierten Patienten}

Patienten mit einem chirurgischen Tracheostoma, z.B. nach Laryngektomie, stellen einen Sonderfall beim Atemwegsmanagement dar. Insbesondere heimbeatmete Patienten sind häufig tracheotomiert und von Pflegekräften vor Ort betreut oder werden vom Rettungsdienst in speziellen Pflegeeinrichtungen angetroffen.

Die häufigste Ursache für eine respiratorische Problematik stellen bei diesen Patienten Verlegungen der einliegenden Trachealkanülen („A-Problem“) dar. Es kann zu Sekretverlegungen oder Verborkungen kommen, was konsekutiv eine Hypoxie bis hin zum Kreislaufstillstand bewirken kann. Das Freimachen bzw. Absaugen von Sekret und ggf. Entfernen der Trachealkanüle und eine Umkanülierung ist eine essenzielle Maßnahme, die auch von nichtärztlichem Rettungsdienstpersonal durchgeführt werden kann [4]. 
Merke

Bei der invasiven Atemwegssicherung und Beatmung tracheotomierter Patienten sollte grundsätzlich eine blockbare Trachealkanüle verwendet werden. Ist keine solche verfügbar, kann auch ein passender Endotrachealtubus (abhängig vom Durchmesser des Tracheostomas, Cave: Einführtiefe beachten) in Notfallsituationen genutzt werden. Beim Wechsel der Trachealkanüle kann mittels eines Führungsstabes per Seldinger-Technik vorgegangen werden.

TAKE HOME MESSAGE

Verlegungen der einliegenden Trachealkanüle stellen die häufigste Problematik bei tracheotomierten Patienten dar. Absaugen von Sekret und ggf. ein Wechsel der Trachealkanüle sind unmittelbar erforderlich, um eine Hypoxie zu vermeiden.

\section{Übernahme beatmeter Patienten im Rettungsdienst}

Insbesondere bei Interhospitalverlegungen (Sekundärtransporte, Intensivverlegung) beatmeter Patienten muss das Rettungsteam nach einer strukturierten Checkliste vorgehen, da es die Verantwortung für den meist intensivpflichtigen Patienten hat. Vor Übernahme bzw. Transportbeginn sollten die Transportfähigkeit überprüft sowie die korrekte Lage sämtlicher Katheter und auch der Atemwege kontrolliert werden. Die routinemäßige Evaluation z. B. nach dem ABCDE-Schema inklusive Auskultation, Dokumentation der Tubustiefe, ggf. Kontrolle und Korrektur der Tubusfixierung sollte strukturiert erfolgen.

TIPP

Bei besonders invasiv beatmeten Patienten empfiehlt sich vor Transportbeginn nach Anschluss an das Transportbeatmungsgerät die Kontrolle der arteriellen Blutgase noch in der abgebenden Klinik. Vor Diskonnektion des Endotrachealtubus kann das kurzzeitige Abklemmen des Beatmungssystems sinnvoll sein, um einen „PEEP-Verlust“ zu minimieren.

Insgesamt sollte auch in zeitkritischen Situationen bei der Übernahme beatmeter Patienten eine sorgfältige Evaluation, Untersuchung und Übergabe erfolgen, um Komplikationen durch den Transport („Transporttrauma“) zu verhindern.

\section{Atemwegsmanagement unter besonderen Bedingungen: Hygiene- maßnahmen bei COVID-19-Verdacht}

In der aktuellen weltweiten COVID-19-Pandemie wurden Empfehlungen zum Atemwegsmanagement von verschiedenen Fachgesellschaften publiziert [25-27].

Ziel aller empfohlenen Maßnahmen ist der Schutz des medizinischen Personals und die Minimierung der Keimexposition. Verschiedene Maßnahmen können die Exposition gegenüber potenziell virushaltigen Aerosolen reduzieren, und es sollten Maßnahmen zur Atemwegssicherung immer unter besonderen Vorkehrungen getroffen werden.

Für die Notfallsituation bei Patienten mit Hinweisen auf eine COVID-19-Infektion (z. B. Husten, Fieber, Dyspnoe, anamnestisch Kontakt zu erkrankten Personen) wird das Anlegen der geeigneten persönlichen Schutzausrüstung (PSA: FFP2-Schutzmaske, Augenschutz, ggf. doppelte Handschuhe, Schutzkittel und Kopfhaube bei invasiven, aerosolbildenden Maßnahmen) empfohlen. Der spontanatmende Patient ohne Sauerstoffbedarf sollte einen Mund-Nasen-Schutz (MNS) tragen.

Cave

Die Durchführung einer nichtinvasiven Beatmung kann zu einer vermehrten Aerosolbildung beitragen.

Eine nichtinvasive Beatmung kann nach strenger Indikationsstellung und Abwägung unter entsprechender PSA des Personals indiziert sein, um den Patienten zunächst zu stabilisieren und z. B. innerklinisch eine invasive Atemwegssicherung zu reevaluieren.

Durchführung: In jedem Fall sollte eine endotracheale Intubation immer unter bestmöglichen Bedingungen erfolgen, um eine potenzielle Kontamination des Teams zu vermeiden. Für die endotracheale Intubation liegen Empfehlungen vor:

- Diese invasive und potenziell aerosolbildende Maßnahme soll unter strenger Indikationsstellung und durch den erfahrensten Anwender durchgeführt werden (hoher First Pass Success).

- Im Rahmen der RSI (unbedingt mit Relaxation zur Verhinderung des Hustenreflexes) sollte möglichst eine Maskenbeatmung vermieden werden.

- Es sollten optimale Vorbereitungen getroffen werden.

- Die Laryngoskopie sollte unbedingt als Videolaryngoskopie und ggf. unter einer transparenten Folie durchgeführt werden [26].

Außerdem sollte auch schon bei der BMV ein geeigneter Filter verwendet werden. 


\section{TAKE HOME MESSAGE}

Zusammenfassend sollten alle Maßnahmen des prähospitalen Atemwegsmanagements unter entsprechender adäquater PSA erfolgen.

Die Indikationsstellung zur invasiven Atemwegssicherung sollte streng erfolgen, allerdings auch nicht zu lange hinausgezögert werden, um das Outcome des Patienten nicht zu verschlechtern. Bei geplanter Atemwegssicherung kann die Anlage eines extraglottischen Atemwegs (EGA) mit konsekutiver endotrachealer Intubation im Verlauf vorteilhaft sein [26].

\section{Ausbildung und Training}

Die rettungsdienstliche als auch notärztliche Ausbildung sehen das Training im Umgang mit Grundlagen der Beatmung, endotracheale Intubationen bzw. die Anwendung von EGA vor [1]. Die empfohlenen Mindestzahlen zur Anwendung von ETI und EGA im Rahmen der Ausbildung sind aktuell vermutlich nicht überall umsetzbar, sollten aber künftig Bestandteil der klinischen Weiterbildung sein. Auch Fortbildungen zum Thema Atemwegsmanagement können ergänzend sinnvoll sein.

Regelmäßige Praxisfortbildungen zum Atemwegsmanagement sind empfohlen, um die Routine zu erhalten und insbesondere die Teamperformance in Notfallsituationen zu optimieren. Hierbei sollten auch das Management von Komplikationen sowie Aspekte der Teamkommunikation integriert werden. Simulationstrainings können nachweislich zu einer Verbesserung der Sicherheit und des Patientenoutcomes beitragen [1,8]. Wünschenswert wären interprofessionelle Simulationstrainings zum Atemwegsmanagement, um insbesondere das Management des schwierigen Atemwegs und von Komplikationen zu beherrschen.

\section{Crew Resource Management bei der Atemwegssicherung}

Neben der Expertise des Teams sind ein strukturierter, ruhiger Ablauf, eine sichere Kommunikation sowie die adäquate Vorbereitung und Antizipation von Komplikationen essenziell, um mitunter letale Verläufe („Cannot intubate, cannot oxygenate“-Situation) zu vermeiden und insgesamt einen sicheren Ablauf im Rahmen des prähospitalen Atemwegsmanagements zu gewährleisten.

Grundregeln des Crew Resource bzw. Team Resource Managements sollten bei jeder prähospitalen Atemwegssicherung beachtet werden. Nach der kritischen Indikationsstellung sollte der Ablauf im Team gemeinsam besprochen werden und eine - auch bei zeitkritischen Situ- ationen - sorgfältige Vorbereitung erfolgen. Die Entscheidung, ob die Atemwegssicherung in der Wohnung des Patienten (sofortige Intervention erforderlich) oder im Rettungswagen (optimierte Bedingungen, gewohnte Umgebung, Material verfügbar, Lagerung des Patienten verbessert) erfolgt, sollte klar im Team abgestimmt werden. Zudem sollten die Induktionsmedikamente mitsamt Dosierungen und das Material im Vorfeld besprochen werden.

Idealerweise sollten sämtliche Gerätschaften und Material, auch für den unerwartet schwierigen Atemweg (EGA, Koniotomieset) sorgfältig nach Checklisten vorbereitet und erst bei vollständiger Vorbereitung die Narkoseinduktion begonnen werden.

\section{TIPPS}

Eine „Closed-Loop-Kommunikation“ mit direkten Rückkopplungen ist essenziell, um gerade in Notfallsituationen Komplikationen, wie z. B. Dosierungsfehler, zu vermeiden.

Auftretende Probleme sollten sofort an das gesamte Team kommuniziert und Alternativen gewählt werden.

Team-Time-out-Regelungen nach dem Prinzip „10 Seconds for 10 Minutes“ können hier hilfreich sein.

Ein strukturiertes Debriefing insbesondere nach dem Auftreten von schwerwiegenden Komplikationen, belastenden Situationen usw. ist zur Qualitätssicherung und persönlichen Verarbeitung hilfreich $[1,8,11]$.

\section{TAKE HOME MESSAGE}

Ein „gelebtes“ Crew Resource Management ist in nahezu allen Notfallsituationen hilfreich, insbesondere bei der Atemwegssicherung kann es aber relevant für das Behandlungsergebnis sein und Sicherheit im Team vermitteln.

\section{Zusammenfassung}

Im Einsatzspektrum des professionellen Rettungsdienstes stellen Atemwegsnotfälle („A-Probleme“) eine seltene und respiratorische Notfälle („B-Probleme“) eine häufige Behandlungsentität dar. Das Atemwegsmanagement hat demnach einen hohen Stellenwert bei allen Notfallbildern, die eine Verlegung der Atemwege und damit eine Atemwegssicherung erfordern. Essenziell ist die Atemwegssicherung auch bei der kardiopulmonalen Reanimation. Notfälle mit Oxygenierungs- und Ventilationsproblemen und erfordern ebenfalls ein Atemwegsmanagement in Form von Sauerstofftherapie und Beatmung. 
Die Vorhaltung entsprechenden Materials, Schulung und regelmäßiges Training, die Etablierung von Standard-Arbeitsanweisungen und gelebtes Teamwork sind wesentliche Aspekte für ein erfolgreiches Atemwegsmanagement. Die zuletzt 2019 aktualisierte S1-Leitlinie „Prähospitales Atemwegsmanagement“ bietet einen praktischen Leitfaden für nahezu sämtliche Aspekte bei der prähospitalen Atemwegssicherung sowohl für pädiatrische als auch erwachsene Patienten.

\section{KERNAUSSAGEN}

- Das prähospitale Atemwegsmanagement ist eine Teamaufgabe und erfordert eine kritische Indikationsstellung zur Atemwegssicherung, eine sorgfältige Vorbereitung und Kommunikation sowie einen gelebten Handlungsalgorithmus zur Bewältigung von Komplikationen wie dem unerwartet schwierigen Atemweg.

- Die S1-Leitlinie „Prähospitales Atemwegsmanagement" bietet wichtige Handlungsempfehlungen für nahezu alle Aspekte der prähospitalen Versorgung und insbesondere für die alternative Atemwegssicherung.

- Ziele des prähospitalen Atemwegsmanagements sind die Sicherstellung einer Normoxie, Normokapnie und eines ausreichenden Aspirationsschutzes.

- Die endotracheale Intubation gilt weiterhin als Goldstandard der invasiven Atemwegssicherung, allerdings kann im Einzelfall davon abgewichen werden.

- Die Beutel-Masken-Beatmung ist als Rückfallebene in allen Notfallsituationen zu sehen, kann aber z. B. bei pädiatrischen Patienten auch als primäres Verfahren sinnvoll sein.

Interessenkonflikt

Erklärung zu finanziellen Interessen

Forschungsförderung erhalten: nein; Honorar/geldwerten Vorteil für Referententätigkeit erhalten: nein; Bezahlter Berater/interner Schulungsreferent/Gehaltsempfänger: nein; Patent/Geschäftsanteile/Aktien (Autor/Partner, Ehepartner, Kinder) an im Bereich der Medizin aktiven Firma: nein; Patent/ Geschäftsanteile/Aktien (Autor/Partner, Ehepartner, Kinder) an zu Sponsoren dieser Fortbildung bzw. durch die Fortbildung in ihren Geschäftsinteressen berührten Firma: nein.

Erklärung zu nichtfinanziellen Interessen

Die Autorinnen/Autoren geben an, dass kein Interessenkonflikt besteht.

\section{Autorinnen/Autoren}

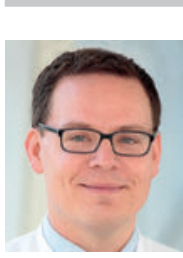

\section{Mark Michael}

Dr. med., Jahrgang 1978. 1999-2006 Studium der Humanmedizin an der Heinrich-Heine-Universität Düsseldorf. Seit 2007 Klinik für Anästhesiologie, Universitätsklinikum Düsseldorf. Seit März 2018 Oberarzt der Zentralen Notaufnahme der Universitätsklinik Düsseldorf. Tätigkeit als Notarzt im Rettungsdienst der Stadt Düsseldorf seit 2008.

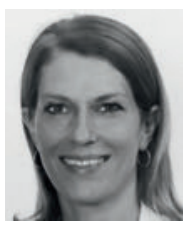

\section{Juliane Tautz}

Dr. med., Jahrgang 1975. 1996-2003 Studium der Humanmedizin an der Medizinischen Hochschule zu Lübeck. Fachärztin für Kinderund Jugendmedizin, Zusatzbezeichnungen Notfallmedizin und Pädiatrische Intensivmedizin. Seit 2017 Oberärztin, Fachabteilung Neonatologische und Pädiatrische Intensivstation, Uniklinik Düsseldorf.

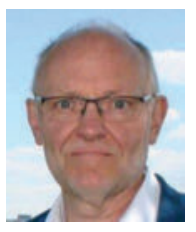

\section{Frank Sensen}

Dr. med., Facharzt für Anästhesie. Seit 2000 Ärztlicher Leiter Rettungsdienst der Landeshauptstadt Düsseldorf.

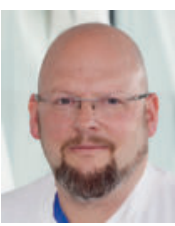

\section{Michael Bernhard}

Privatdozent Dr. med., Jahrgang 1976, MHBA, Facharzt für Anästhesiologie, Zusatzbezeichnung Notfallmedizin, Klinische Akut- und Notfallmedizin und Intensivmedizin. Habilitation 2014. Seit 2018 Ärztlicher Leiter der Zentralen Notaufnahme, Universitätsklinikum Düsseldorf. Wissenschaftliche Schwerpunkte: Atemwegsmanagement und konservatives Schockraummanagement in der Zentralen Notaufnahme.

\section{Korrespondenzadresse}

PD Dr. med. Michael Bernhard, MHBA

Zentrale Notaufnahme

Universitätsklinikum Düsseldorf

Heinrich-Heine-Universität Düsseldorf

Moorenstraße 5

40225 Düsseldorf

Deutschland

michael.bernhard@med.uni-duesseldorf.de

Wissenschaftlich verantwortlich gemäß Zertifizierungsbestimmungen

Wissenschaftlich verantwortlich gemäß Zertifizierungsbestimmungen für diesen Beitrag ist PD Dr. med. Michael Bernhard, Düsseldorf. 
[1] Timmermann A, Böttiger BW, Byhahn C et al. S1-Leitlinie: Prähospitales Atemwegsmanagement. AWMF Online 2019. AWMF-Registernummer 001-040. Im Internet (Stand: 27.10.2020): https://www.awmf.org/leitlinien/detail/I//001040.html

[2] Bernhard M, Bein B, Böttiger BW et al. Handlungsempfehlung zur prähospitalen Notfallnarkose beim Erwachsenen. AWMFRegisternummer 001/030. Stand März 2015. Im Internet (Stand: 27.10.2020): https://www.awmf.org/uploads/ tx_szleitlinien/001-030l_S1_Praehospitale_Notfallnarkose_Erwachsene_2015-03-verlaengert.pdf

[3] Lechleuthner A. Der Pyramidenprozess - die fachliche Abstimmung der invasiven Maßnahmen im Rahmen der Umsetzung des Notfallsanitätergesetzes. Notarzt 2014; 30: 112-117

[4] Muster-Algorithmen 2020 zur Umsetzung des Pyramidenprozesses im Rahmen des NotSanG. Deutscher Berufsverband Rettungsdienst (DBRD) e.V., Version 5.0. Im Internet (Stand: 27.10.2020): https://www.dbrd.de/aktuell/aktuelles/492dbrd-muster-algorithmen-2020-online

[5] Indikationskatalog für den Notarzteinsatz. Handreichung für Telefondisponenten in Notdienstzentralen und Rettungsleitstellen, Stand: 22.02.2013. Dtsch Arztebl 2013; 110: A-521/ B-465/C-465

[6] Reifferscheid F, Harding U. Einheitliche Notarztqualifikation: Wie steht es 13 Jahre nach der Einführung um die Zusatzweiterbildung Notfallmedizin? Anästh Intensivmed 2017; 58: 4451

[7] Piepho T, Cavus E, Noppens R et al. S1-Leitlinie Atemwegsmanagement. Anaesthesist 2015; 64: 859-873. doi:10.1007/ s00101-015-0087-6

[8] Grande B, Kolbe M, Biro P. Difficult airway management and training: simulation, communication, and feedback. Curr Opin Anesthesiol 2017, 30: 743-747

[9] Soar J, Nolan JP, Böttiger BW et al. European Resuscitation Council Guidelines for Resuscitation 2015. Resuscitation 2015; 95: 100-147

[10] Westhoff M, Schönhofer B, Neumann P et al.; Deutsche Gesellschaft für Pneumologie und Beatmungsmedizin, Hrsg. S3Leitlinie Nichtinvasive Beatmung als Therapie der akuten respiratorischen Insuffizienz. AWMF-Registernummer 020/004, 2015. Im Internet (Stand: 27.10.2020): https://www.awmf. org/leitlinien/detail/II/020-004.html

[11] Scott JA, Heard SO, Zayaruzny M et al. Airway management in critical illness - an update. Chest 2020; 157: 877-887

[12] Lupton JR, Schmicker RH, Stephens S et al. Outcomes with the use of bag-valve-mask ventilation during out-of-hospital cardiac arrest in the pragmatic airway resuscitation trial. Acad Emerg Med 2020; 27: 5. doi:10.1111/acem. 13927

[13] Soar ], Donnino M, Maconochie I et al. 2018 International consensus on cardiopulmonary resuscitation and emergency cardiovasvular care science with treatment recommendations summary. Resuscitation 2018; 133: 194-206

[14] Deutsche Gesellschaft für Unfallchirurgie, Hrsg. S3-Leitlinie Polytrauma/Schwerverletztenbehandlung. AWMF-Registernummer 012/19. 2016. Im Internet (Stand: 27.10.2020): https://www.awmf.org/uploads/tx_szleitlinien/012019I_S3_Polytrauma_Schwerverletzten-Behandlung_201708.pdf

[15] Brown CA, Kaji AH, Fantegrossi A et al. Video laryngoscopy compared to augmented direct laryngoscopy in adult emergency department tracheal intubations: A National Emergency Airway Registry (NEAR) Study. Acad Emerg Med 2020; 27: 2. doi:10.1111/acem.13851

[16] Bernhard M, Bax SN, Hartwig T et al. Airway Management in the Emergency Department (The OcEAN-Study) - a prospective single centre observational cohort study. Scand J Trauma Resusc Emerg Med 2019; 27: 20

[17] Özkurtul O, Struck MF, Fakler J et al. Physician-based on-scene airway management in severely injured patients and in-hospital consequences: is the misplaced intubation an underestimated danger in trauma management? Trauma Surg Acute Care Open 2019; 4: e000271

[18] Price TM, McCoy EP. Emergency front of neck access in airway management. Brit J Anaesth 2019; 19: 246-253. doi:10.1016/ j.bjae.2019.04.002

[19] Fischer M, Wnent J, Gräsner JT et al. Jahresbericht des Deutschen Reanimationsregisters - Außerklinische Reanimation 2019. Anästh Intensivmed 2020; 61: V89-V93

[20] Behrens NH, Fischer M, Krieger T et al. Effect of airway management strategies during resuscitation from out-of-hospital cardiac arrest on clinical outcome: A registry-based analysis. Resuscitation 2020; 152: 157-164

[21] Benger JR, Kibry K, Black S et al. Effect of a Strategy supraglottic airway device vs. tracheal intubation during out-of-hospital cardiac arrest on functional outcome. The AIRWAY-2 randomized clinical trial. JAMA 2018; 320: 779-791

[22] Jabre P, Penaloza A, Pinero D et al. Effect of bag-mask ventilation vs. endotracheal intubation during cardiopulmonary resuscitation on neurological outcome after out-of-hospital cardiopulmonary arrest. A randomized clinical trial. JAMA 2018; 319: 779-787

[23] Wang HE, Schmicker RH, Daya MR et al. Effect of a strategy of initial laryngeal tube insertion vs. endotracheal intubation on 72-hour survival in adults with out-of-hospital cardiac arrest a randomized clinical trial. JAMA 2018; 320: 769-778

[24] Wölfl C, Bouillon B, Lackner C et al. Prehospital Trauma Life Support ${ }^{\circledR} \quad$ PHTLS $\left.^{\circledR}\right)$. Unfallchirurg 2008; 111: 688-694. doi:10.1007/s00113-008-1466-0

[25] Kluge S, Janssens U, Welte T et al. S1-Leitlinie: Empfehlungen zur intensivmedizinischen Therapie von Patienten mit COVID-19 (Version 2; 19.06.2020). AWMF-Registernummer 113/001. Im Internet (Stand: 27.10.2020): https://www.dgiin.de/files/dgiin/leitlinien/20200619_S1-Leitlinie_Empfehlungen-intensivmedizinische-Therapie-COVID-19.pdf

[26] Schälte G, Kehl F, Didion N et al. Besonderheiten des Atemwegsmanagements bei Patienten mit vermuteter oder gesicherter COVID-19 Erkrankung und bei Patienten ohne Infektion während der Corona-Pandemie. Empfehlungen von DGAI und BDA. Anästh Intensivmed 2020; 61: S132-S136. doi:10.19224/ai2020.S132

[27] European Resuscitation Council. COVID-19-Leitlinien des European Resuscitation Council. 24.04.2020. Im Internet (Stand: 27.10.2020): https://www.dgiin.de/files/dgiin/leitlinien/ 202005_ERC_Leitlinien_Covid-19_Deutsch.pdf 
Zum Weiterlesen und Vertiefen

[1] Böhm SH, Kremeier P, Tusman G et al. Volumetrische Kapnographie zur Analyse und Optimierung von Ventilation und Gasaustausch. Anaesthesist 2020; 69: 361-270. doi:10.1007/ s00101-020-00747-0

[2] Carlson JN, Wang HE. Updates in emergency airway management. Curr Opin Crit Care 2018; 24: 525-530

[3] Ilper $\mathrm{H}$, Kunz $\mathrm{T}$, Faist $\mathrm{M}$ et al. Nichtinvasive Evaluation der Atemwege vor Sedierungen oder Narkosen. Update im Rahmen der Überarbeitung der S1-Leitlinie „Atemwegsmanagement“ 2015 der DGAl. Anaesthesist 2020; 69: 521-532

[4] Michael M, Hossfeld B, Häske D et al. Analgesie, Sedierung und Anästhesie in der Notfallmedizin. Anästh Intensivmed 2020; 61: 51-65
Bibliografie

Notfallmedizin up2date 2020; 15: 357-378

DOI $10.1055 / \mathrm{a}-1154-1959$

ISSN $1611-6550$

(c) 2020. Thieme. All rights reserved.

Georg Thieme Verlag KG, Rüdigerstraße 14, 70469 Stuttgart, Germany 
Diese Fortbildungseinheit ist in der Regel 12 Monate online für die Teilnahme verfügbar.

Den genauen Einsendeschluss finden Sie unter https://eref.thieme.de/CXEWPFH.

Sollten Sie Fragen zur Online-Teilnahme haben, finden Sie unter https://cme.thieme.de/hilfe

eine ausführliche Anleitung. Wir wünschen viel Erfolg beim Beantworten

der Fragen!

Unter https://eref.thieme.de/CXEWPFH oder über den QR-Code kommen Sie direkt zur Startseite des Wissenstests.

VNR 2760512020158724845

\section{Frage 1}

Eine der folgenden Aussage zur nichtinvasiven Ventilation (NIV) trifft nicht zu. Welche?

A Die CPAP-Therapie (= continuous positive Airway Pressure) ist definiert als Spontanatmung mit Anwendung eines PEEP (= positive endexpiratory Pressure).

B Bei hyperkapnischem Lungenversagen ist die NIV-Therapie mit inspiratorischer Druckunterstützung indiziert.

C Bei hypoxischem Lungenversagen ist die CPAP-Therapie mit PEEP-Anwendung indiziert.

D Notfallsanitäter sind nicht befugt, eine nichtinvasive Ventilation durchzuführen, da sie in der Handhabung nicht ausgebildet sind.

E Unterstützend kann eine Analgosedierung z. B. mit Opioiden sinnvoll sein.

\section{Frage 2}

Welche Notfallsituation stellt am ehesten eine Indikation zur Notfallkoniotomie dar?

A anaphylaktische Reaktion mit ausgeprägter Ödembildung bei massiver Zungenschwellung (Mundöffnung nicht möglich) mit „Cannot Ventilate, Cannot Intubate“-Situation

B Patient unter Reanimationsmaßnahmen, zweimalige Laryngoskopie frustran, Beutel-Masken-Beatmung möglich

C Patient mit kardialem Lungenödem unter NIV-Therapie

D Kleinkind mit Schädel-Hirn-Trauma und erschwerten Intubationsbedingungen, aber problemloser Beutel-Masken-Beatmung

E Patient mit V.a. intrakranielle Blutung, zwei frustrane Intubationsversuche, Larynxtubus problemlos zu etablieren

\section{Frage 3}

Verschiedene Maßnahmen sind geeignet, die Beutel-MaskenBeatmung zu optimieren. Welche gehört nicht dazu?

A Anwendung des „doppelten C-Griffs“

B Nutzung eines Oropharyngealtubus (z. B. Guedel-Tubus)

C Entfernen der Zahnprothesen

D leichte Reklination des Kopfes

E Unterpolsterung des Kopfes („verbesserte Jackson-Position“)

\section{Frage 4}

Welche Zuordnung nach dem „DOPES“-Schema trifft nicht zu?

A D: Dislokation des Endotrachealtubus durch fehlerhafte Konnektion des Beatmungssystems

B O: Obstruktion bei ausgeprägtem Bronchospasmus nach Intubation

C P: Pulslosigkeit nach Narkoseeinleitung

D E: defekte Ausrüstung (Equipment), z. B. falsch konnektierter Beatmungsschlauch

E S: Magenüberblähung (Stomach) nach Maskenbeatmung mit der Notwendigkeit zur Entlastung, z.B. durch eine Magensonde

\section{Frage 5}

Bei einem 2-jährigen Kleinkind $(15 \mathrm{~kg})$ sind Reanimationsmaßnahmen nach einer Fremdkörperaspiration erforderlich, nach etwa 5 Minuten wird ein ROSC (Return of spontaneous Circulation) detektiert. Welche Atemwegssicherung sollte bei noch bestehender Bradypnoe bis zur Übernahme in der Klinik (Transportzeit: 4 Minuten) bei geringer Expertise in der Intubation bei Kindern vorgenommen werden?

A Versuch der endotrachealen Intubation (Goldstandard) nach Narkoseeinleitung

B assistierte bzw. kontrollierte Beatmung mit dem Beatmungsbeutel (BMV)

C Anlage eines Larynxtubus ohne Narkoseinduktion

D Einbringen einer Larynxmaske nach Induktion mit 5 mg Midazolam i.v.

E Notfallkoniotomie bei stattgehabter Atemwegsverlegung

\section{Frage 6}

Welche Indikation zur invasiven Atemwegssicherung besteht beim Traumapatienten?

A Amputation rechte Hand bei Kreißsägenverletzung, Patient ABCD-stabil, GCS 15

B Verbrennung ca. 10\% der Körperoberfläche am Rücken, ABstabil, Schocksymptomatik

C schweres Schädel-Hirn-Trauma, GCS 5, Anisokorie, ABC-stabil

D Messerstichverletzung abdominell, „C-Problem“ mit Schocksymptomatik, GCS 15

E kindliches Trauma: Kind 4 Jahre, aus 3 Metern Höhe gestürzt, ABC-stabil, GCS 14 
Punkte sammeln auf CME. thiemede

Fortsetzung $\ldots$

\section{Frage 7}

Mit welcher Maßnahme kann bei einem bewusstlosen Patienten am effektivsten ein fester Fremdkörper im Bereich des Larynx entfernt werden?
A Heimlich-Manöver
B Schläge auf den Rücken
C manuelle Ausräumung
D Absaugung mit großlumigen Absaugkathetern
E Entfernung mittels Laryngoskopie und Magill-Zange

\section{Frage 8}

Sie werden als Notarzt zu einem 62-jährigen Patienten gerufen, der bewusstlos im Bett liegend vorgefunden wurde. Bei GCS 7 und Anisokorie stellen Sie den Verdacht auf eine intrakranielle Blutung. Respiratorisch und hämodynam ist der Patient stabil, Schutzreflexe sind vorhanden. Bei der Untersuchung fällt allerdings eine verminderte Mundöffnung (ca. $2 \mathrm{~cm}$ ) nach einer kieferchirurgischen Versorgung auf. Eine Therapielimitierung liegt nicht vor. Die maximalversorgende Klinik ist ca. 5 Minuten Transportzeit entfernt. Welches Vorgehen bezüglich der Atemwegssicherung erscheint adäquat?

A Versuch der konventionellen Intubation vor Ort nach Induktion mit Propofol, Fentanyl und Rocuronium

B Narkoseinduktion und Versuch der Beutel-Masken-Ventilation

C primäre Durchführung einer Notfallkoniotomie

D Oxygenierung mittels Sauerstoffinhalation (15 I/min via Inhalationsmaske mit Reservoir, Voranmeldung im Schockraum mit Anforderung des Anästhesieteams und dem Hinweis auf Vorhaltung einer fiberoptischen Intubation

E Narkoseinduktion ohne Muskelrelaxierung und Versuch der Etablierung einer Larynxmaske, da in dieser Situation zwingend eine invasive Atemwegssicherung notwendig ist.

\section{Frage 9}

Welcher der folgenden Befunde ist kein sicheres Zeichen einer endotrachealen Intubation?

A Nachweis eines et $\mathrm{CO}_{2}$ von $42 \mathrm{mmHg}$ nach Anschluss der Kapnografie

B Intubation unter laryngoskopischer bzw. videolaryngoskopischer Sicht

C Detektion von etCO $\mathrm{CO}_{2}$ im Farbindikator („Farbumschlag“)

D seitengleiche Thoraxexkursionen bei manueller Beatmung

E erneute Lagekontrolle mittels Laryngoskop und Verifizierung der Tubuslage endotracheal

\section{Frage 10}

Bei welchem der folgenden Befunde besteht keine Indikation zur nichtinvasiven Beatmung?

A hyperkapnisches Lungenversagen bei akut-exazerbierter COPD (chronisch obstruktive Lungenerkrankung)

B palliative Situation bei Bronchialkarzinom, nun Hypoxie bei Pneumonie

C Hypoxie bei unklarer Vigilanzminderung, GCS 8 mit fehlenden Schutzreflexen

D akute respiratorische Insuffizienz bei hypertensivem Lungenödem

E infektexazerbierte COPD, V.a. ambulant erworbene Pneumonie 